\title{
Two-dimensional ferroelectric tunnel junction: the case of monolayer In:SnSe/SnSe/Sb:SnSe homostructure
}

\author{
Xin-Wei Shen ${ }^{1}$, Yue-Wen Fang ${ }^{1,2 *}$, Bo-Bo Tian ${ }^{1}$, Chun-Gang Duan ${ }^{1,3 *}$ \\ ${ }^{1}$ State Key Laboratory of Precision Spectroscopy and \\ Key Laboratory of Polar Materials and Devices, \\ Ministry of Education, \\ Department of Optoelectronics, \\ East China Normal University, \\ Shanghai, 200241, China \\ ${ }^{2}$ Department of Materials Science and Engineering, \\ Kyoto University, Kyoto 606-8501, Japan \\ ${ }^{3}$ Collaborative Innovation Center of Extreme Optics, \\ Shanxi University, Taiyuan, \\ Shanxi 030006, China
}




\begin{abstract}
Ferroelectric tunnel junctions, in which ferroelectric polarization and quantum tunneling are closely coupled to induce the tunneling electroresistance (TER) effect, have attracted considerable interest due to their potential in non-volatile and low-power consumption memory devices. The ferroelectric size effect, however, has hindered ferroelectric tunnel junctions from exhibiting robust TER effect. Here, our study proposes doping engineering in a two-dimensional in-plane ferroelectric semiconductor as an effective strategy to design a two-dimensional ferroelectric tunnel junction composed of homostructural $p$-type semiconductor/ferroelectric/n-type semiconductor. Since the in-plane polarization persists in the monolayer ferroelectric barrier, the vertical thickness of two-dimensional ferroelectric tunnel junction can be as thin as monolayer. We show that the monolayer In:SnSe/SnSe/Sb:SnSe junction provides an embodiment of this strategy. Combining density functional theory calculations with non-equilibrium Green's function formalism, we investigate the electron transport properties of In:SnSe/SnSe/Sb:SnSe and reveal a giant TER effect of $1460 \%$. The dynamical modulation of both barrier width and barrier height during the ferroelectric switching are responsible for this giant TER effect. These findings provide an important insight towards the understanding of the quantum behaviors of electrons in materials at the twodimensional limit, and enable new possibilities for next-generation non-volatile memory devices based on flexible two-dimensional lateral ferroelectric tunnel junctions.
\end{abstract}

*Electronic address: fyuewen@gmail.com (Y.-W.F); cgduan@clpm.ecnu.edu.cn (C.-G.D) 


\section{INTRODUCTION}

The past decades have witnessed an explosion in the field of ferroelectric materials [1-3], headlined by the design of ferroelectric tunnel junctions (FTJs) with the aim of accelerating their commercial applications into non-volatile information devices [4-14]. FTJs are composed of two metallic electrodes separated by a thin ferroelectric barrier. The information is encoded via the non-volatile ferroelectric polarization that can be electrically switched. Switching the polarization gives rise to dramatic change of the tunneling electroresistance (i.e.TER effect [4]), making it possible to non-destructively readout the polarization state that carries information. The increasing suppression of ferroelectricity by the depolarization field as ferroelectric materials are reduced down to nanometers $[15,16]$, or in other words, the ferroelectric size effect [17], however, has impeded the development of nanometer-size FTJs. Although several widely studied ferroelectric oxides (e.g., $\mathrm{BaTiO}_{3}$ ) have been experimentally proved to show switchable polarization down to the thickness of 1-4 unit cells [18-21], the lack of stability and reproducibility at room temperature of FTJs based on these conventional ferroelectric thin films cannot keep up with the ever-growing commercial needs for the ultra-low-power, high-speed, and non-volatile nanoscale memory devices.

In spite of the difficulties in growing thin films of ferroelectric oxides due to their high demand for the growth conditions [22-24], some early first-principles studies have predicted the existence of in-plane ferroelectricity in monolayer binary inorganic compounds [25, 26]. These works have prompted the active search for the two-dimensional (2D) ferroelectrics

both in theory and experiment [27-39]. Among these studies, the group-IV chalcogenide semiconductors have shown to be one class of the most promising 2D ferroelectric materials. As reported in the experimental study by Chang et al., atomic-thick SnTe exhibits nearroom-temperature in-plane ferroelectricity, and the ferroelectricity in ultrathin SnTe films above 2 unit cells can persist above room temperature [27]. A nonvolatile in-plane ferroelectric random access memory (FeRAM) based on 3 unit cells SnTe is designed in Chang et al.'s study to readout information non-destructively, which is more superior than conventional FeRAM where reading is destructive [27]. In addition to the 2D monochalcogenide SnTe, the 2D trichalcogenide $\alpha-\operatorname{In}_{2} \mathrm{Se}_{3}$, in which out-of-plane and in-plane polarization are intrinsically intercorrelated, can sustain the ferroelectricity up to $700 \mathrm{~K}$ [37]. By integrating 2D $\alpha$ - $\mathrm{In}_{2} \mathrm{Se}_{3}$ into a ferroelectric Schottky diode junction, high current density of $\sim 12 \mathrm{~A} / \mathrm{cm}^{2}$ 
is reported to be more than two times of the conventional ferroelectric diode junctions [36]. The high-quality monolayer SnTe and $\alpha-\mathrm{In}_{2} \mathrm{Se}_{3}$ can be prepared by molecular beam epitaxial technique or physical vapor deposition [27, 36, 40], indicating the feasibility of synthesis of 2D ferroelectric materials. These recent successes of maintaining stable electric polarization in 2D semiconductors, which are both from an experimental as well as from a theoretical perspective, motivate us to explore their interesting properties and potential applications in ferroelectric non-volatile memories based on 2D-FTJs.

Herein, through doping engineering in 2D semiconductors to establish electrodes ( $i$.e., $p$-type and $n$-type semiconductors), in combination with the coupling between the robust in-plane ferroelectricity and quantum tunneling in ferroelectric semiconducting barrier at the two-dimensional limit, we theoretically design a new class 2D-FTJs based on homostructure. Using a model which takes into account screening of polarization charges in electrodes, charge accumulation/depletion at semiconductor/ferroelectric interfaces, reversible metallization of the ferroelectric barrier [41], and direct quantum tunneling across a ferroelectric barrier, we demonstrate the possibility to obtain giant TER effect in 2D-FTJ. As an example 2DFTJ, In:SnSe/SnSe/Sb:SnSe homostructure is investigated by first-principles calculations with non-equilibrium Green's function formalism. We find a giant TER effect of 1460\%, which is on account of a dual modulation of both barrier width and barrier height. To our knowledge, this is the first demonstration of the 2D-FTJ based on homostructure that holds promise in future memory devices.

The geometry optimizations and electronic structure calculations of slab models are performed within density-functional theory (DFT) using the projector augmented wave (PAW) method [42], as implemented in the Vienna ab initio Simulation Package (VASP) [43, 44]. The exchange correlation functional is treated in generalized gradient approximation (GGA) with the type of Perdew-Burke-Ernzerhof (PBE) [45]. The kinetic-energy cutoff of $500 \mathrm{eV}$ is applied to the plane wave expansion and a $\Gamma$-centered $1 \times 12 \times 1 \mathbf{k}$ points grid is adopted for Brillouin zone sampling. All the structures are optimized until the Hellmann-Feynman forces are below $1 \mathrm{meV} / \AA$, and the convergence threshold of electronic energy is $10^{-6} \mathrm{eV}$. A vacuum space of $15 \AA$ is used to avoid interactions between adjacent layers. The spontaneous ferroelectric polarization $\left(P_{s}\right)$ is determined by the Berry phase method $[46,47]$.

The device properties of the 2D-FTJ are calculated using density functional theory plus non-equilibrium Green's function formalism (DFT+NEGF approach) [48, 49] as imple- 
mented in Atomistix ToolKit-Virtual NanoLab (ATK-VNL) software package [50]. Double$\zeta$ plus polarization basis set is employed, and a real-space mesh cut-off energy of 80 Hartree is used to guarantee the good convergence of the device configuration. The electron temperature is set at $300 \mathrm{~K}$. The $1 \times 21 \times 101 \mathbf{k}$ mesh is used for the self-consistent calculations to eliminate the mismatch of Fermi level between electrodes and the central region. An increased number 201 of $\mathbf{k}$ points along $y$ axis is adopted during the calculations of transmission spectra and spatially resolved device density of states.

\section{RESULTS AND DISCUSSION}

Different from the architecture of conventional vertical FTJs built on heterostructures [51], the 2D-FTJ in our study makes the utmost of the structural features of 2D ferroelectrics. We take 2D ferroelectric group-IV monochalcogenide (e.g., SnSe [28] or SnTe [27]) as an example to elucidate our model device. As illustrated in Figure 1, a pure 2D ferroelectric monochalcogenide semiconductor is used as the ferroelectric barrier, on the other hand, its hole and electron doped forms ( $i$.e., $p$-type and $n$-type semiconductors) work as the respective left and right electrodes concurrently. Hence, this new class of 2D-FTJs $p$-semiconductor/ferroelectric/ $n$-semiconductor $(p$-SC/FE $/ n$-SC) are architected on homostructures in avoid of laminating several different materials into heterostructures as the conventional FTJs, which can be expected to reduce the difficulty in device manufacture.

In Thomas-Fermi theory, the screening length of a metallic material is defined as

$$
\delta=\frac{1}{e} \sqrt{\frac{\varepsilon}{\rho}}
$$

where $\varepsilon$ is the dielectric permittivity and $\rho$ is the density of states at the Fermi level $E_{\mathrm{F}}$. In the homostructural 2D-FTJ displayed in Figure 1, the dielectric permittivity of left/right electrode is equivalent to that of pure 2D ferroelectric group-IV monochalcogenide at saturation polarization [52]. Hence, the screening lengths of the two electrodes only depend on their densities of states at the $E_{\mathrm{F}}$ that can be easily controlled by doping. In order to introduce our model easily, we assume $\delta_{1}<\delta_{2}$, in which $\delta_{1}$ and $\delta_{2}$ are the screening lengths of left and right electrodes, respectively. The model in which $\delta_{1}>\delta_{2}$ can also be found in Supporting Information.

Figure 2(a) and (b) schematically depict the charge distributions and charge density 


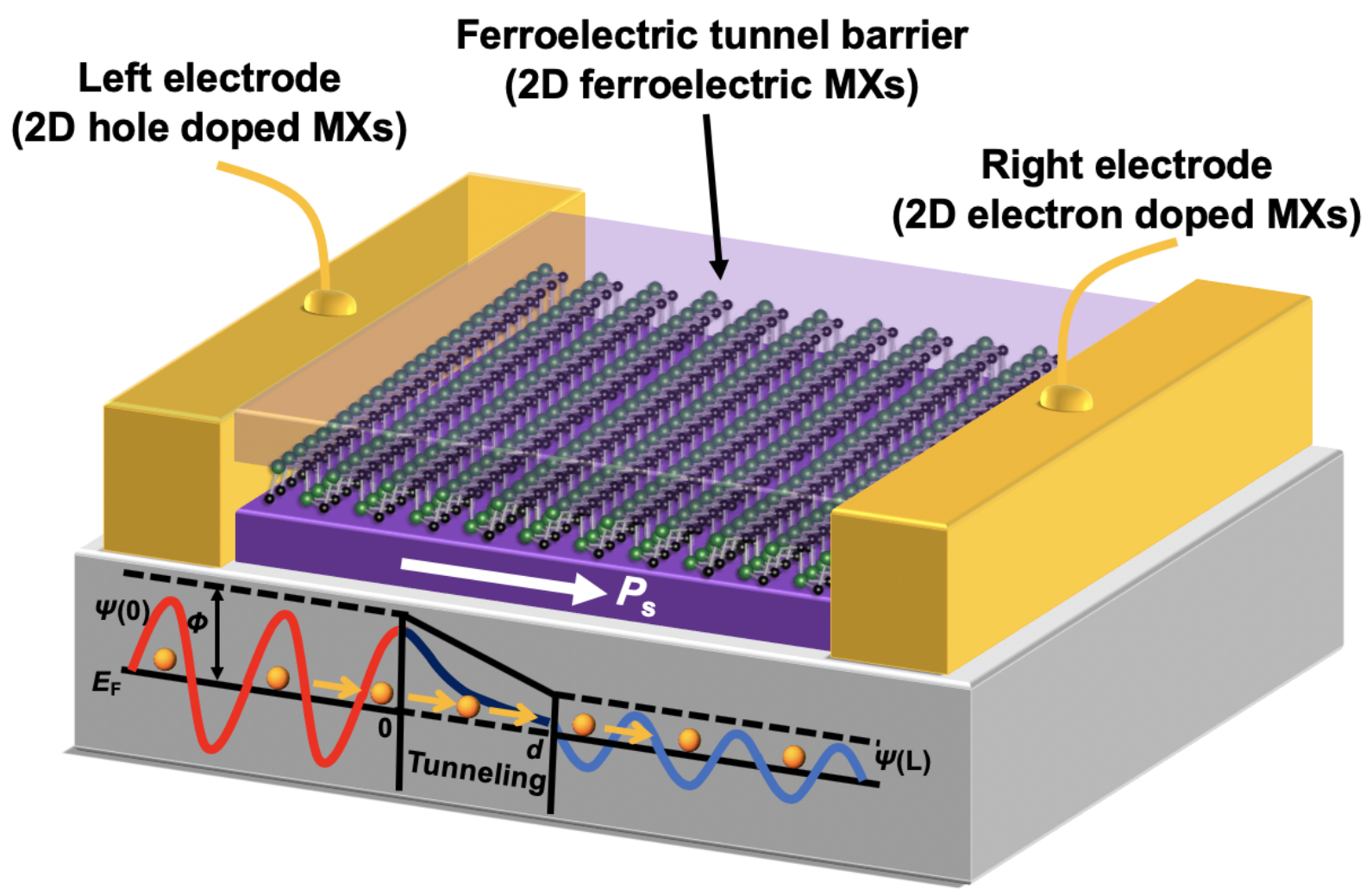

FIG. 1: Schematic diagram of a two-dimensional ferroelectric tunnel junction (2D-FTJ) device based on homostructure. A pure 2D monochalcogenide (MX) semiconductor with in-plane ferroelectricity serves as a tunneling barrier. The left and right electrodes are obtained by $p$-type and $n$-type doping of the same MX. The inset illustrates the wave-particle duality of the quantum tunneling and the change of barrier $\Phi$ in response to the polarization reversal. Governed by quantum mechanics, the electrons tunnel across the barrier in the form of evanescent state which decays exponentially through the barrier in amplitude.

profiles in the 2D-FTJ, where $d$ is the original width of 2D ferroelectric barrier. When polarization is rightward (referred to as $P_{+x}$ state), negative bound charge $\left(-P_{\mathrm{bc}}\right)$ and positive ferroelectric bound charges $\left(+P_{\mathrm{bc}}\right)$ are introduced at the left edge and right edge of the ferroelectric barrier, respectively. In this case, the hole (electron) carriers in the electrode of $p$-SC ( $n$-SC) bbbecome accumulated owning to the spontaneous electric field at the interface (i.e., ferroelectric polarization field effect). On the contrary, the carriers in both $p$-SC and $n$-SC electrodes are depleted as the polarization is leftward (referred to as $P_{-x}$ state). In order to investigate the change of barrier in response to the ferroelectric 
switching quantitatively, we adopt a Thomas-Fermi screening mode [4]. In this way, the electrostatic potentials within left and right electrodes can be written as:

$$
\varphi_{i}= \pm \frac{P_{s} d \delta_{i}}{\varepsilon_{0} \varepsilon_{i}\left[\varepsilon_{\mathrm{FE}}\left(\frac{\delta_{1}}{\varepsilon_{1}}+\frac{\delta_{2}}{\varepsilon_{2}}\right)+d\right]}, i=1 \text { or } 2,
$$

where $i=1$ for left electrode $p$-SC, $i=2$ for right electrode $n$-SC, $\varepsilon_{0}$ is the permittivity of free space, $\varepsilon_{\mathrm{FE}}$ is the relative permittivity of the ferroelectric layer, and $\varepsilon_{i}$ is the dielectric permittivity. The sign '+' ('-') corresponds to the polarization pointing to (away from) the studied electrode. Using Equation 2 and the assumption of $\delta_{1}<\delta_{2}$, we can compare the electrostatic potential energy at $p$-SC/FE and $\mathrm{FE} / n$-SC interfaces:

$$
\left|e \varphi_{1}\right| \equiv|e \varphi(0)|<\left|e \varphi_{2}\right| \equiv|e \varphi(d)|
$$

We set the charge of $e$ to '-1' in Equation 3 for the simplification of model analysis, it becomes $\left|\varphi_{1}\right|<\left|\varphi_{2}\right|$. This leads to an asymmetry in the electrostatic potential energy profiles for the opposite polarization directions, which can be seen in Figure 2(c). In addition to the electrostatic potential energy, we note that the tunneling electrons should also overcome electronic potential energy and potential energy barrier inside 2D ferroelectric material. As pointed out by the earlier studies $[4,53]$ the potential barriers can be assumed to be a rectangular shape of height $U$ with respect to the Fermi level $E_{\mathrm{F}}$. Thus, the average barrier height in either $P_{+x}$ or $P_{-x}$ state can be given as

$$
\left\{\begin{array}{l}
\Phi_{R}=U+\left(\varphi_{1}-\varphi_{2}\right) / 2 \\
\Phi_{L}=U+\left(\varphi_{2}-\varphi_{1}\right) / 2
\end{array}\right.
$$

where subscripts $R$ and $L$ correspond to $P_{+x}$ and $P_{-x}$ states, respectively. Setting Equation 3 into Equation 4 yields

$$
\Phi_{R}<\Phi_{L}
$$

indicating the average barrier height in $P_{-x}$ state is higher than that in $P_{+x}$ state. The resulted barrier heights in these two states are comparatively illustrated in Figure 2(d).

Besides it follows from Figure 2(d) that the effective barrier width can also be changed by the polarization switching. As pointed out by some recent experimental and theoretical studies [54-57], off-center displacements and metallic conductivity can coexist in doped ferroelectrics. In our 2D-FTJ model, we consider such a condition where the introduced dopants 
in the electrodes will not eliminate the polar distortions completely, i.e., the electrodes enter polar metallic states. In addition, we note that charge leakage can be induced at the interface when a polar metallic material is interfaced with a ferroelectric material [41, 58]. For the $P_{+x}$ state of 2D-FTJ, carriers are accumulated at the $p$-SC/FE and $n$-SC/FE interfaces. This leads to the enhancement of the charge leakage, and therefore the regions of ferroelectric barrier near the two interfaces may become conductive, resulting in reduction of the effective tunneling width. We refer this decreased tunnelling width to as $d_{1}$ which is smaller than the original width $d$ of the ferroelectric barrier, as can be seen from the left panel of Figure 2(d). By contrast, carriers are depleted at the two interfaces in $P_{-x}$ state, The majority carriers will be partially cancelled out near the semiconductor/ferroelectric interfaces, only leaving the immobile ionized acceptors and donors [53], as shown in the right panel of Figure 2(a). Under this condition, charge leakage of $P_{-x}$ state is weaker than $P_{+x}$ state. As a result, some ferroelectric barrier regions near interfaces will transform back into insulating state, and the band alignment in $P_{-x}$ state is also changed with respect to $P_{+x}$ state. If the charge leakage is sufficiently weak, all the ferroelectric barrier regions and even partial regions in the electrodes may become insulating [41]. In this case, the effective barrier width is increased to $d_{2}$, which is larger than effective barrier width $d_{1}$ of the $P_{+x}$ state. The modulation of effective barrier width is also known as the "reversible metallization of the barrier" as reported by Liu et al. [41]. It is due to the ferroelectric dual modulation of both barrier width and barrier height in 2D-FTJ, the tunneling electrons in the $P_{-x}$ state have to overcome additional barrier height and barrier width. Since the tunneling conductance is determined by the barrier height and barrier width [4], the conductance of $P_{+x}$ state will be dramatically enhanced compared to $P_{-x}$ state. The higher conductance state is conventionally referred to as "ON" state, with a comparison of the lower conductance state referred to as "OFF" state, as displayed in Figure 2(d). We note that in conventional FTJs like Metal-1/ferroelectric/Metal-2 heterostructures [6], only barrier height is generally modified by polarization switching. Therefore, the 2D-FTJs in our study can be expected to realize enhanced TER effect.

In order to realize the functional $2 \mathrm{D}-\mathrm{FTJ} p$-SC/FE$/ n$-SC, we turn to study real materials. SnSe is a 2D monochalcogenide with robust in-plane ferroelectric polarization above room temperature, as pointed out by Ref. [28]. The crystal structure of monolayer SnSe is explicitly provided in the Supporting Information, In the $x y$ plane, the ferroelectric polar- 

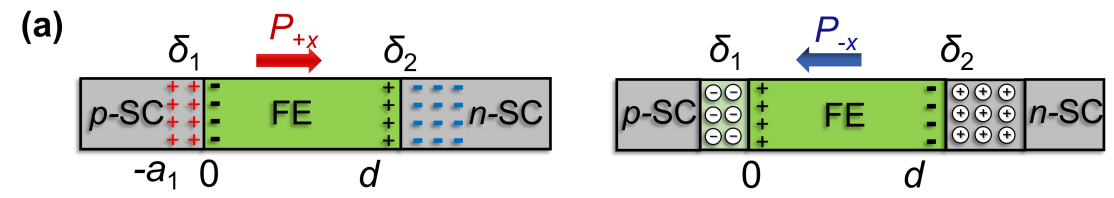

(b)
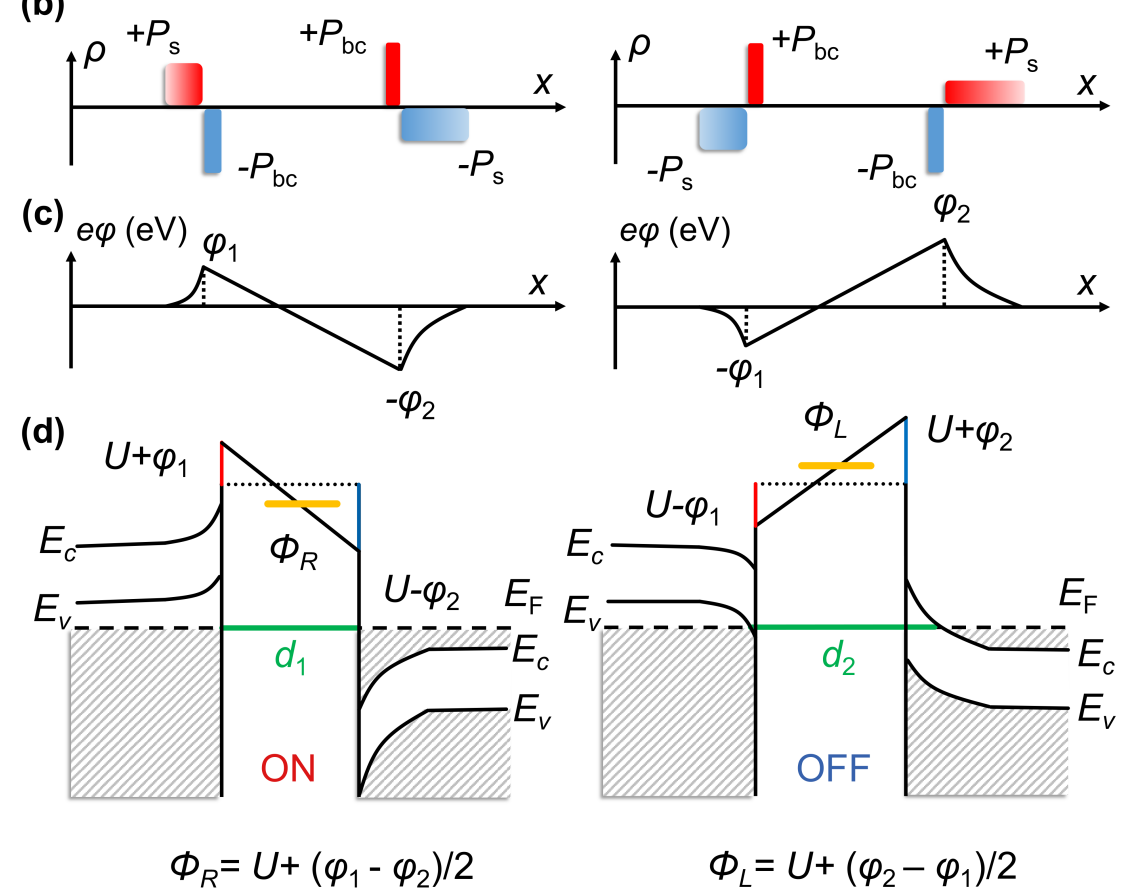

FIG. 2: Switching mechanisms of the 2D-FTJ $p$-SC/FE/n-SC. (a) The respective schematics of 2D-FTJ in $P_{+x}$ (left panel) and $P_{-x}$ (right panel) states. The black "+" and "-" symbols in the ferroelectric barrier region represent positive and negative ferroelectric bound charges, respectively. The red "+" in $p$-SC and blue "_" in $n$-SC electrodes represent hole and electrons, respectively. The " $\oplus$ " and " $\ominus$ " represent ionized donors and acceptors respectively. (b) The distributions of charge densities. (c) Electrostatic potential energy profiles. (d) The overall potential energy profiles with corresponding band diagrams. The barrier width in either $P_{+x}$ or $P_{-x}$ state is given by the green line. The average potential barrier height is indicated by the short orange line. The Fermi level, as shown by black dashed line, is set as the reference of the barrier height.

ization along $x$ and $y$ axis are equivalent due to the symmetry [59], and we will only discuss the case along $x$-direction throughout the study. Using Berry phase approach [46, 47], we have revisited its polarization in the monolayer limit and found it is $\sim 1.82 \times 10^{-10} \mathrm{C} / \mathrm{m}$, in agreement with previous study [28]. In the Supporting Information, we also revisit the double-well potential energy profile of monolayer SnSe and its lattice dynamics properties. 
As many other ferroelectric materials like bulk $\mathrm{BaTiO}_{3}$ and $\mathrm{PbTiO}_{3}$ [60], monolayer SnSe also features a typical symmetric potential profile [59], i.e., the phase transition between its paraelectric and ferroelectric states is continuous and spontaneous below a critical temperature. Utilizing different valences of elements, In (Sb) is doped into monolayer SnSe to form $p$-type ( $n$-type) semiconductor. The experimental feasibility of such doped SnSe is discussed in Supporting Information. In our study, the doped carrier concentration in In:SnSe or Sb:SnSe reaches up to $6.2 \times 10^{20} \mathrm{~cm}^{-3}$ because the size of unit cell In:SnSe or $\mathrm{Sb}: \mathrm{SnSe}$ are composed of 4-unit cells $\mathrm{SnSe}$ where one $\mathrm{Sn}$ atom is replaced by one $\mathrm{In}$ or $\mathrm{Sb}$ atom [59]. By examining the electronic structures of $p$-type In:SnSe and $n$-type Sb:SnSe [59], the In and Sb dopants are found to introduce shallow levels. Hence, the doped holes and electrons are free to move in materials. In addition, we find the density of states of In:SnSe is about two times of that of Sb:SnSe, indicating the screening length of In:SnSe is smaller than that of Sb:SnSe according to Thomas-Fermi theory (details are provided in Supporting Information). Therefore, In:SnSe and Sb:SnSe can act as semiconductor electrodes of a 2D-FTJ. We then set up a In:SnSe/SnSe/Sb:SnSe homostructure as a implementation of $p$-SC/FE/n-SC 2D-FTJ. In this In:SnSe/SnSe/Sb:SnSe homostructure, the region of ferroelectric SnSe is a stacking of 18 unit-cells along [100] direction $(\sim 8 \mathrm{~nm})$, and In:SnSe (Sb:SnSe) plays as the $p$-type ( $n$-type) semiconductor electrode. The optimized atomic structures of In:SnSe/SnSe/Sb:SnSe homostructure in the $P_{+x}$ and $P_{-x}$ states are displayed in Figure 3(a). In addition to the robust ferroelectric displacements in the barrier region, we find the polar displacements remain in the In:SnSe and $\mathrm{Sb}$ :SnSe regions. In contrast to the conventional Metal-1/ferroelectric/Metal-2 FTJ [6] or all-oxide FTJ [61] in which lattice mismatch is inevitably generated by the electrodes and ferroelectrics due to their unmatched lattice constants, our In:SnSe/SnSe/Sb:SnSe homostructure completely eliminates the lattice constant mismatch and will not introduce abrupt structure distortions at the ferroelectric/electrode interfaces. In addition, the ferroelectric polarization of monolayer SnSe in this 2D-FTJ is spontaneous, which avoids using sophisticated chemical methods [29, 62] to maintain stable and switchable 2D ferroelectricity.

The stability and robustness of in-plane ferroelectricity in a 2D ferroelectric barrier sandwiched between two doped electrodes lies at the heart of 2D-FTJ device. To verify the in-plane ferroelectricity of monolayer SnSe barrier survives in the monolayer In:SnSe/SnSe/Sb:SnSe homostructure, we compute the total energy of the homostructure as 

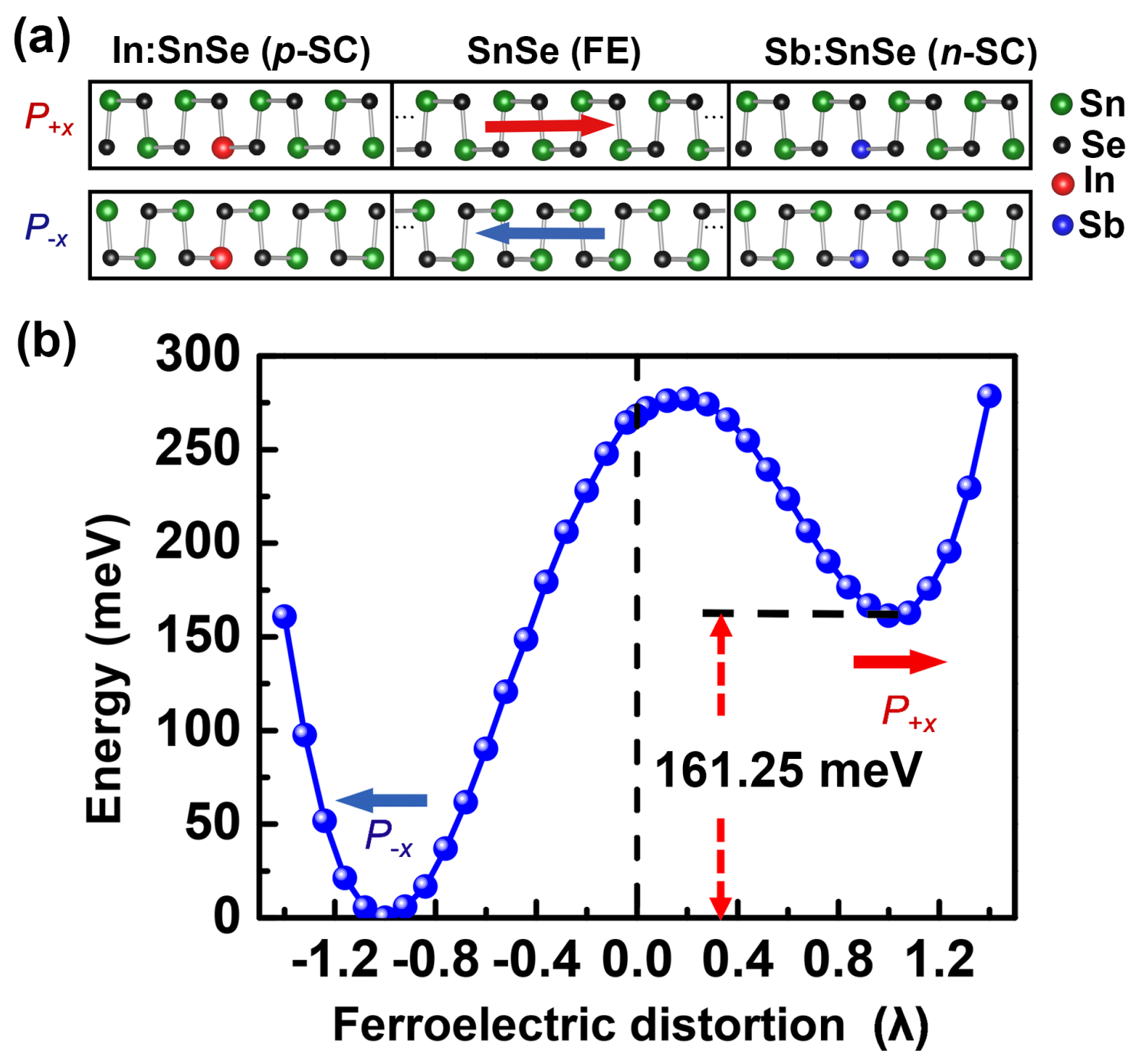

FIG. 3: (a) The calculated atomic structure and the schematic diagram (shaded regions) of 2DFTJ In:SnSe/SnSe/Sb:SnSe. The red and blue arrows indicate $P_{+x}$ and $P_{-x}$, respectively. Only a few unit cells are shown for illustrating the ferroelectric barrier region and electrode regions because of the page width limitations. The lengths along $x$-axis in $P_{+x}$ and $P_{-x}$ states are both $114 \AA$. (b) Calculated asymmetric potential energy profile as a function of ferroelectric distortions in the 2D-FTJ In:SnSe/SnSe/Sb:SnSe. The ferroelectric displacements $\lambda$ along [100] directions are normalized so that $\lambda=+1$ and -1 correspond to the $P_{+x}$ and $P_{-x}$ states, respectively. The energy is summed up to all the atoms in the structure. The energy of $P_{-x}$ state is set as the reference.

a function of normalized polar displacements $\lambda$. As shown in Figure 3(b), a double-well potential profile is observed, indicating the stability of ferroelectricity in the monolayer SnSe. This can be ascribed to the small depolarization field of $6.5 \times 10^{6} \mathrm{~V} / \mathrm{m}$ in SnSe barrier, 
which can be well screened by the the left/right electrodes (see Supporting Information for more details). Different from a free-standing SnSe monolayer with a symmetric potential profile [59], the monolayer In:SnSe/SnSe/Sb:SnSe homostructure displays an asymmetric potential profile: 1$)$ the energy minima at $\lambda=-1$ (i.e., $P_{-x}$ state) and $\lambda=1\left(P_{+x}\right.$ state $)$ are inequivalent with energy difference of $161.25 \mathrm{meV} /$ homostructure; 2) the energy maximum corresponding to a paraelectric phase is approximately located at $\lambda=0.2$, which slightly deviates from $\lambda=0$. This asymmetry is a consequence of the symmetry breaking introduced by the two different dopants in the two electrodes. The asymmetry can also be observed from the polar displacements profile in Supporting Information. These results indicate the barriers at the two interfaces are also asymmetric, and will be responsible for the TER effect [63].

In order to evaluate the performance of 2D-FTJ In:SnSe/SnSe/Sb:SnSe, density functional theory plus non-equilibrium Green's function formalism is used to study the electrical conductance and TER effect. The device configurations for $P_{+x}$ and $P_{-x}$ states are explicitly shown in Figure 4(a). The left/right extension layer (buffer layer) is as wide as around $35 \AA$, which is confirmed to be large enough to screen the electrostatic potential [59]. In our calculations, the transmission coefficients and reflection matrices are determined by matching the wave functions of the scattering region with linear combinations of propagating Bloch states in the electrodes. Since the electronic states at the $E_{\mathrm{F}}$ dominate the transport properties, the zero-bias electrical conductance within the Landauer-Büttiker formula [64] can be evaluated as:

$$
G=G_{0} \sum_{k_{\|}} T\left(E_{\mathrm{F}}, k_{\|}\right) .
$$

where $G_{0}=2 e^{2} / h$ is the conductance quantum, $e$ is the electron charge, $h$ is the Planck's constant, and $T\left(E_{\mathrm{F}}, k_{\|}\right)$is the transmission coefficient at the Fermi energy for a given Bloch wave vector $k_{\|}=\left(k_{x}, k_{y}\right)$ in the $2 \mathrm{D}$ Brillouin zone. By integrating the transmission probability for states at the Fermi energy over the 2D Brillouin zone, total conductance $(G)$ can be calculated. In the $P_{+x}$ state, $G_{R}=1.003 \times 10^{-9} \mathrm{~S}$, by contrast in the $P_{-x}$ state, $G_{L}=$ $6.435 \times 10^{-11} \mathrm{~S}$. Following the conventional definition in previous study [61], the TER ratio in our study is defined as:

$$
\mathrm{TER}=\frac{G_{R}-G_{L}}{G_{L}} .
$$

As a result, the reversal of ferroelectric polarization in the 2D-FTJ In:SnSe/SnSe/Sb:SnSe 
leads to a significantly enhanced TER effect at zero bias, which is approximately about $1460 \%$. This TER effect is about one order larger than those in conventional all-oxide FTJs such as $\mathrm{LaNiO}_{3} / \mathrm{BaTiO}_{3} / \mathrm{LaNiO}_{3}[63]$ and $\mathrm{SrRuO}_{3} / \mathrm{BaTiO}_{3} / \mathrm{SrRuO}_{3}$ [61] at zero bias. Note that the migrations of electrons or holes near the semiconductor surfaces are nearly ignored during the transport calculations in which only electron cloud diffusion is taken into account, making the change of barrier width underestimated by ferroelectric polarization reversal. Hence, the actual TER effect in experiment should be even higher than the theoretical value [53]. In addition, although the band gap of semiconductor is usually underestimated in DFT calculations, we find the correction of band gap of ferroelectric barrier does not affect the TER effect of the studied 2D-FTJ significantly [59], which is similar to the conventional FTJs [65].

In order to understand the large change in the conductance ratio during the polarization reversal, the $k_{\|}$-resolved transmissions at $E_{\mathrm{F}}$ are shown in Figure 4(b). In the $P_{+x}$ state, the transmission coming from the two blue stripe regions (around $k_{y}=+0.42 /-0.42$ ) of the 2D Brillouin zone are largest, indicating the feature of resonant tunneling. We find the transmission eigenstates around this region show much smaller decay rate than those around $\Gamma$ point, which is responsible for the significant tranmission. The discussions of transmission eigenstates are available in the Supporting information. Compared to the $P_{+x}$ state, the transmission in the $P_{-x}$ state is largely reduced, leading to lower conductance than the $P_{+x}$ state. This explains the observed giant TER effect in the monolayer In:SnSe/SnSe/Sb:SnSe homostructure.

Finally, we compare the effective barriers for the two polarization states in the 2D-FTJ In:SnSe/SnSe/Sb:SnSe. As mentioned above, the combination of DFT-calculated density of states and Thomas-Fermi theory indicates the screening length of left electrode In:SnSe is smaller than the right electrode $\mathrm{Sb}: \mathrm{SnSe}$, this indicates the assumption that $\delta_{1}<\delta_{2}$ in the model of Figure 2 is indeed true in our studied case. We can then get $\Phi_{R}<\Phi_{L}$ through Equations 3 and 4, i.e., the barrier height for $P_{+x}$ state is smaller than that for $P_{-x}$ state. In order to understand the change of barrier width, we study the electronic structure across the 2D-FTJ In:SnSe/SnSe/Sb:SnSe device by carrying out the analysis of real-space device DOS (DDOS) projected onto the device $x$-axis. The corresponding results for the two polarization states are displayed in Figure 5. We find that the band diagrams calculated by DFT + NEGF approach are qualitatively consistent with those obtained in our model in 

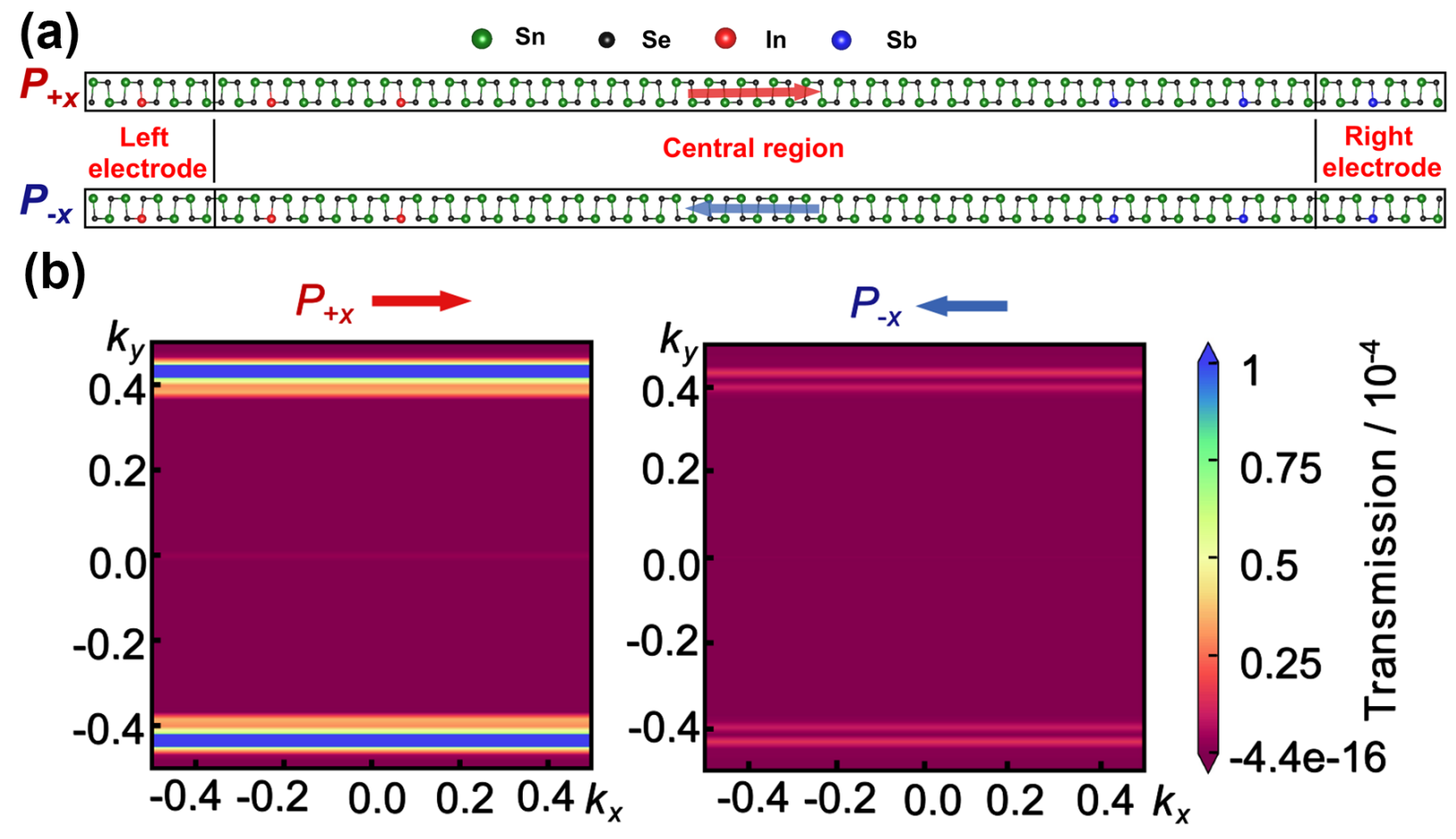

FIG. 4: (a) The device configurations in the DFT+NEGF calculations for $P_{+x}$ and $P_{-x}$ states. (b) The $k_{\|}$-resolved transmissions in 2D Brillouin zone at the Fermi energy through the 2D-FTJ In:SnSe/SnSe/Sb:SnSe for $P_{+x}$ and $P_{-x}$ states. Polarization directions are shown by arrows. The $k_{\|}$-resolved transmissions for $P_{+x}$ and $P_{-x}$ states use the same scale as given by the color bar.

Figure 2. In particular, barrier width in the $P_{-x}$ state is indeed increased compared to the $P_{+x}$ state owing to the interfacial metallization of the edge regions in ferroelectric barrier controlled by the ferroelectric switching. In Figure 5, we have used red solid rectangles to highlight the effective tunneling regions, in which the evolution of the valence band maximum along $x$-axis is guided by the red dashed line. The built-in electric field caused by the work function step can be clearly observed through the tilting of bands in SnSe [66]. When the ferroelectric polarization is pointing to the right (i.e., $P_{+x}$ state), the depolarizing field is parallel to the built-in electric field, and hence the band edges in SnSe is tilted. On the other hand, the bands of SnSe become slightly flat since the depolarization field is antiparallel to the built-in field in the $P_{-x}$ state. More details about the reversible metallization of the monolayer SnSe barrier can be found in the layer-resolved density of states provided in Supporting Information. Therefore, in addition to the raised barrier height as polarization is flipped from $P_{+x}$ to $P_{-x}$ state, the barrier width is also increased. This makes the electron 

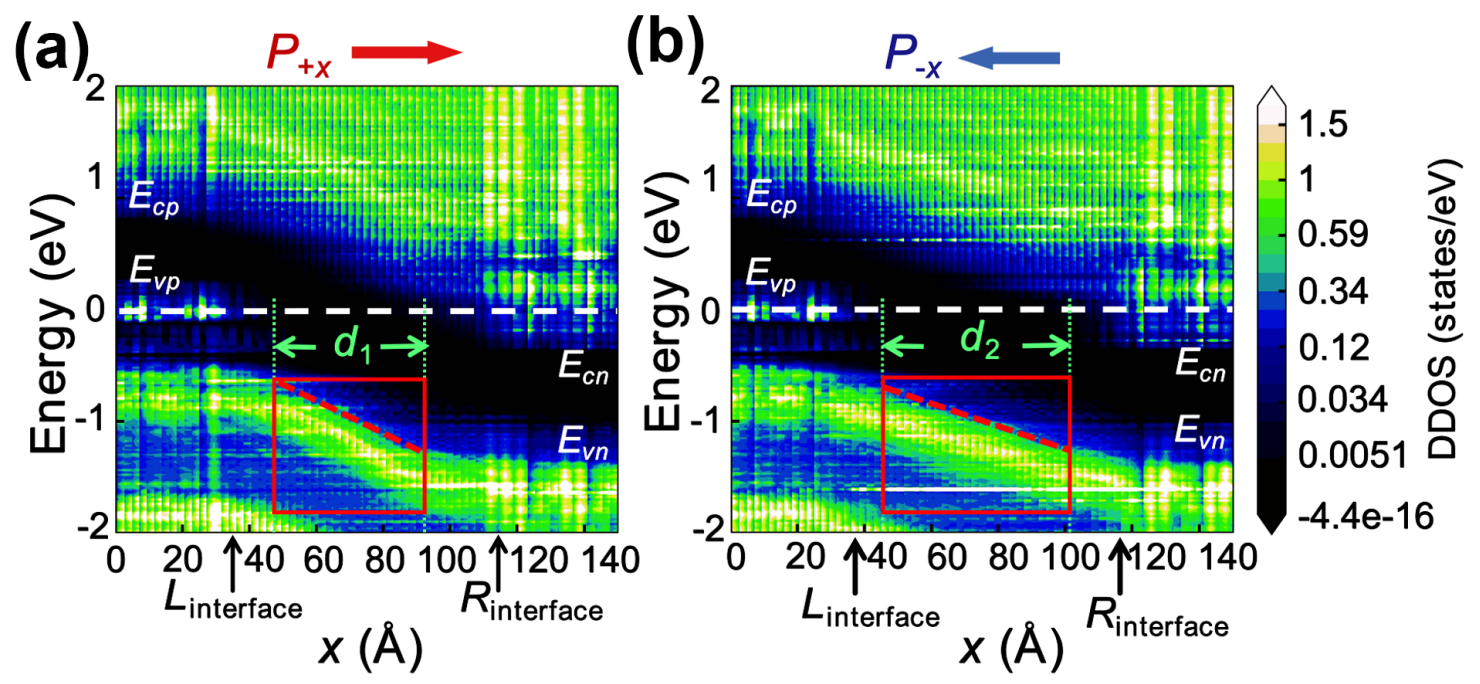

FIG. 5: The device density of states (DDOS) of the 2D-FTJ In:SnSe/SnSe/Sb:SnSe device projected onto its $x$-axis. (a) $P_{+x}$ state. (b) $P_{-x}$ state. The abscissa is the Cartesian coordinate of central region along the $x$-axis. The color bar on the right indicates the DOS amplitude. $E_{c i}$ and $E_{v i}$ are the conduction band minimum and valence band maximum of the electrodes, respectively. The Fermi level is set to zero, which is shown by the white dashed line. The interface of semiconductor/ferroelectric is initially set to be located around $35 \AA$ and $114 \AA$. The black arrow indicates the interface between 2D ferroelectric SnSe and electrode In:SnSe (Sb:SnSe). The red rectangle represents the region of effective ferroelectric tunneling barrier, and the red dashed lines are used to guide the evolution of the valence band maximum along $x$-axis. The green arrow indicates the width of the tunneling barrier. The black regions in the tunneling regions indicate the band gap of ferroelectric barrier.

tunneling much easier in $P_{+x}$ state than in $P_{-x}$ state, which is account for the observed giant TER effect.

\section{CONCLUSIONS}

In summary, we have proposed a method to design two-dimensional ferroelectric tunnel junction based on planar $p$-type semiconductor/ferroelectric/ $n$-type semiconductor homostructure via the doping engineering in a two-dimensional ferroelectric semiconductor with in-plane electric polarization. Combining density functional theory calculations with 
non-equilibrium Green's function formalism, a giant TER effect of $1460 \%$ is observed in our newly designed 2D-FTJ In:SnSe/SnSe/Sb:SnSe homostructure, which is comparable to that of conventional all-oxide FTJs. The tunable tunneling barrier width that is generally absent in conventional FTJs, as well as the tunneling barrier height, is responsible for the enhanced TER effect. The dynamical modulation of barrier width stems from the depletion/accumulation of majority carriers near the semiconductor surface in response to the reversal of ferroelectricity. SnSe is thought to be the most flexible in known 2D atomic materials [67], hence monolayer In:SnSe/SnSe/Sb:SnSe homostructures can be promising memory blocks in the wearable devices and artificial synapses, which has larger advantages over the conventional FTJs. The proposed strategy in our study is applicable to design novel 2D-FTJs using other 2D ferroelectric materials. We hope this work will stimulate the experimental endeavors of fabricating 2D-FTJs with giant TER effect to accelerate their commercial applications into ultra-low-power, high-speed, and non-volatile nanoscale memory devices.

\section{Supporting Information}

The Supporting Information can be found after the main text. It is also available free of charge on the ACS Publications website at DOI: 10.1021/acsaelm.9b00146.

- The overall potential energy profile if $\delta_{1}>\delta_{2}$; in-plane ferroelectricity in monolayer SnSe; carrier concentration in doped SnSe; phonon dispersions; dopants with shallow levels; screening lengths in electrodes; experimental feasibility; depolarization field; polar displacements profile; layer-resolved density of states; transmission eigenstate; width of extension layer; Hubbard $U$ effect

\section{acknowledgement}

This work was supported by the National Key R\&D Program of China (2017YFA0303403), the National Natural Science Foundation of China (Grant No. 11774092, 51572085) and Shanghai Science and Technology Innovation Action Plan (No. 17JC1402500). Computations were performed at the ECNU computing center. 
Competing interests: The authors declare no competing financial or non-financial interests.

[1] S. V. Kalinin, A. N. Morozovska, L. Q. Chen, and B. J. Rodriguez, Rep. Prog. Phys. 73, $056502(2010)$.

[2] C. Bowen, H. Kim, P. Weaver, and S. Dunn, Energy \& Environ. Sci. 7, 25 (2014).

[3] L. W. Martin and A. M. Rappe, Nat. Rev. Mater. 2, 16087 (2017).

[4] M. Y. Zhuravlev, R. F. Sabirianov, S. S. Jaswal, and E. Y. Tsymbal, Phys. Rev. Lett. 94, $246802(2005)$.

[5] E. Y. Tsymbal and H. Kohlstedt, Science 313, 181 (2006).

[6] J. P. Velev, C.-G. Duan, K. D. Belashchenko, S. S. Jaswal, and E. Y. Tsymbal, Phys. Rev. Lett. 98, 137201 (2007).

[7] J. Scott, Nat. Mater. 6, 256 (2007).

[8] V. Garcia, S. Fusil, K. Bouzehouane, S. Enouz-Vedrenne, N. D. Mathur, A. Barthelemy, and M. Bibes, Nature 460, 81 (2009).

[9] V. Garcia, M. Bibes, L. Bocher, S. Valencia, F. Kronast, A. Crassous, X. Moya, S. EnouzVedrenne, A. Gloter, D. Imhoff, et al., Science 327, 1106 (2010).

[10] A. Chanthbouala, V. Garcia, R. O. Cherifi, K. Bouzehouane, S. Fusil, X. Moya, S. Xavier, H. Yamada, C. Deranlot, N. D. Mathur, et al., Nat. Mater. 11, 860 (2012).

[11] C. Lu, W. Hu, Y. Tian, and T. Wu, Appl. Phys. Rev. 2, 021304 (2015).

[12] W. J. Hu, Z. Wang, W. Yu, and T. Wu, Nat. Commun. 7, 10808 (2016).

[13] S. Boyn, J. Grollier, G. Lecerf, B. Xu, N. Locatelli, S. Fusil, S. Girod, C. Carrétéro, K. Garcia, S. Xavier, et al., Nat. Commun. 8, 14736 (2017).

[14] W. Huang, Y.-W. Fang, Y. Yin, B. Tian, W. Zhao, C. Hou, C. Ma, Q. Li, E. Y. Tsymbal, C.-G. Duan, et al., ACS Appl. Mater. Interfaces 10, 5649 (2018).

[15] J. Junquera and P. Ghosez, Nature 422, 506 (2003).

[16] N. A. Spaldin, Science 304, 1606 (2004).

[17] S. Li, J. A. Eastman, J. M. Vetrone, C. M. Foster, R. E. Newnham, and L. E. Cross, Jpn. J. Appl. Phys. 36, 5169 (1997).

[18] D. D. Fong, G. B. Stephenson, S. K. Streiffer, J. A. Eastman, O. Auciello, P. H. Fuoss, and 
C. Thompson, Science 304, 1650 (2004).

[19] D. A. Tenne, A. Bruchhausen, N. D. Lanzillotti-Kimura, A. Fainstein, R. S. Katiyar, A. Cantarero, A. Soukiassian, V. Vaithyanathan, J. H. Haeni, W. Tian, et al., Science 313, $1614(2006)$.

[20] D. A. Tenne, P. Turner, J. D. Schmidt, M. Biegalski, Y. L. Li, L. Q. Chen, A. Soukiassian, S. Trolier-McKinstry, D. G. Schlom, X. X. Xi, et al., Phys. Rev. Lett. 103, 177601 (2009).

[21] P. Maksymovych, M. Huijben, M. Pan, S. Jesse, N. Balke, Y.-H. Chu, H. J. Chang, A. Y. Borisevich, A. P. Baddorf, G. Rijnders, et al., Phys. Rev. B 85, 014119 (2012).

[22] K. J. Choi, M. Biegalski, Y. L. Li, A. Sharan, J. Schubert, R. Uecker, P. Reiche, Y. B. Chen, X. Q. Pan, V. Gopalan, et al., Science 306, 1005 (2004).

[23] D. D. Fong, A. M. Kolpak, J. A. Eastman, S. K. Streiffer, P. H. Fuoss, G. B. Stephenson, C. Thompson, D. M. Kim, K. J. Choi, C. B. Eom, et al., Phys. Rev. Lett. 96, 127601 (2006).

[24] H. Lu, X. Liu, J. D. Burton, C.-W. Bark, Y. Wang, Y. Zhang, D. J. Kim, A. Stamm, P. Lukashev, D. A. Felker, et al., Adv. Mater. 24, 1209 (2012).

[25] S. N. Shirodkar and U. V. Waghmare, Phys. Rev. Lett. 112, 157601 (2014).

[26] D. Di Sante, A. Stroppa, P. Barone, M.-H. Whangbo, and S. Picozzi, Phys. Rev. B 91, 161401 (2015).

[27] K. Chang, J. Liu, H. Lin, N. Wang, K. Zhao, A. Zhang, F. Jin, Y. Zhong, X. Hu, W. Duan, et al., Science 353, 274 (2016).

[28] R. Fei, W. Kang, and L. Yang, Phys. Rev. Lett. 117, 097601 (2016).

[29] M. Wu and X. C. Zeng, Nano Lett. 16, 3236 (2016).

[30] M. Wu, S. Dong, K. Yao, J. Liu, and X. C. Zeng, Nano letters 16, 7309 (2016).

[31] T. Hu, H. Wu, H. Zeng, K. Deng, and E. Kan, Nano Lett. 16, 8015 (2016).

[32] R. Haleoot, C. Paillard, T. P. Kaloni, M. Mehboudi, B. Xu, L. Bellaiche, and S. Barraza-Lopez, Phys. Rev. Lett. 118, 227401 (2017).

[33] H. Wang and X. Qian, 2D Mater. 4, 015042 (2017).

[34] W. Ding, J. Zhu, Z. Wang, Y. Gao, D. Xiao, Y. Gu, Z. Zhang, and W. Zhu, Nat. Commun. 8, 14956 (2017).

[35] C. Huang, Y. Du, H. Wu, H. Xiang, K. Deng, and E. Kan, Phys. Rev. Lett. 120, 147601 (2018).

[36] S. M. Poh, S. J. R. Tan, H. Wang, P. Song, I. H. Abidi, X. Zhao, J. Dan, J. Chen, Z. Luo, 
S. J. Pennycook, et al., Nano Lett. 18, 6340 (2018).

[37] J. Xiao, H. Zhu, Y. Wang, W. Feng, Y. Hu, A. Dasgupta, Y. Han, Y. Wang, D. A. Muller, L. W. Martin, et al., Phys. Rev. Lett. 120, 227601 (2018).

[38] C. Cui, W.-J. Hu, X. Yan, C. Addiego, W. Gao, Y. Wang, Z. Wang, L. Li, Y. Cheng, P. Li, et al., Nano Lett. 18, 1253 (2018).

[39] J. Liu and S. T. Pantelides, 2D Mater. 6, 025001 (2019).

[40] J. Zhou, Q. Zeng, D. Lv, L. Sun, L. Niu, W. Fu, F. Liu, Z. Shen, C. Jin, and Z. Liu, Nano Lett. 15, 6400 (2015).

[41] X. Liu, J. D. Burton, and E. Y. Tsymbal, Phys. Rev. Lett. 116, 197602 (2016).

[42] P. E. Blöchl, Phys. Rev. B 50, 17953 (1994).

[43] G. Kresse and J. Furthmüller, Phys. Rev. B 54, 11169 (1996).

[44] G. Kresse and D. Joubert, Phys. Rev. B 59, 1758 (1999).

[45] J. P. Perdew, K. Burke, and M. Ernzerhof, Phys. Rev. Lett. 77, 3865 (1996).

[46] R. D. King-Smith and D. Vanderbilt, Phys. Rev. B 47, 1651 (1993).

[47] R. Resta, Rev. Mod. Phys. 66, 899 (1994).

[48] J. Taylor, H. Guo, and J. Wang, Phys. Rev. B 63, 245407 (2001).

[49] M. Brandbyge, J.-L. Mozos, P. Ordejón, J. Taylor, and K. Stokbro, Phys. Rev. B 65, 165401 (2002).

[50] Atomistix ToolKit version 2014.3-Virtual NanoLab version 2017.2, QuantumWise A/S (www.quantumwise.com).

[51] V. Garcia and M. Bibes, Nat. Commun. 5, 4289 (2014).

[52] Y. Wang, X. Liu, J. D. Burton, S. S. Jaswal, and E. Y. Tsymbal, Phys. Rev. Lett. 109, 247601 (2012).

[53] Z. Wen, C. Li, D. Wu, A. Li, and N. Ming, Nat. Mater. 12, 617 (2013).

[54] H. J. Zhao, A. Filippetti, C. Escorihuela-Sayalero, P. Delugas, E. Canadell, L. Bellaiche, V. Fiorentini, and J. Íñiguez, Phys. Rev. B 97, 054107 (2018).

[55] F. Cordero, F. Trequattrini, F. Craciun, H. T. Langhammer, D. A. B. Quiroga, and P. S. Silva, Phys. Rev. B 99, 064106 (2019).

[56] J.-x. Gu, K.-j. Jin, C. Ma, Q.-h. Zhang, L. Gu, C. Ge, J.-s. Wang, C. Wang, H.-z. Guo, and G.-z. Yang, Phys. Rev. B 96, 165206 (2017).

[57] T. Kolodiazhnyi, M. Tachibana, H. Kawaji, J. Hwang, and E. Takayama-Muromachi, Phys. 
Rev. Lett. 104, 147602 (2010).

[58] Y.-W. Fang and H. Chen, arXiv preprint arXiv:1901.08771 (2019).

[59] See the Supporting Information for more details.

[60] Y.-W. Fang, H.-C. Ding, W.-Y. Tong, W.-J. Zhu, X. Shen, S.-J. Gong, X.-G. Wan, and C.-G. Duan, Sci. Bull. 60, 156 (2015).

[61] J. P. Velev, C.-G. Duan, J. Burton, A. Smogunov, M. K. Niranjan, E. Tosatti, S. Jaswal, and E. Y. Tsymbal, Nano Lett. 9, 427 (2008).

[62] Q. Yang, W. Xiong, L. Zhu, G. Gao, and M. Wu, J. Am. Chem. Soc. 139, 11506 (2017).

[63] L. L. Tao and J. Wang, Appl. Phys. Lett. 108, 062903 (2016).

[64] R. Landauer, Philos. Mag. 21, 863 (1970).

[65] L. L. Tao and J. Wang, J. Appl. Phys. 119, 224104 (2016).

[66] G. Gerra, A. K. Tagantsev, and N. Setter, Phys. Rev. Lett. 98, 207601 (2007).

[67] L.-C. Zhang, G. Qin, W.-Z. Fang, H.-J. Cui, Q.-R. Zheng, Q.-B. Yan, and G. Su, Sci. Rep. 6, 19830 (2016). 


\title{
Supporting Information for "Two-dimensional ferroelectric tunnel junction: the case of monolayer In:SnSe/SnSe/Sb:SnSe homostructure"
}

\author{
Xin-Wei Shen ${ }^{1}$, Yue-Wen Fang ${ }^{1,2 *}$, Bo-Bo Tian ${ }^{1}$, Chun-Gang Duan ${ }^{1,3 *}$ \\ ${ }^{1}$ State Key Laboratory of Precision Spectroscopy and \\ Key Laboratory of Polar Materials and Devices, \\ Ministry of Education, \\ Department of Optoelectronics, \\ East China Normal University, \\ Shanghai, 200241, China \\ ${ }^{2}$ Department of Materials Science and Engineering, \\ Kyoto University, Kyoto 606-8501, Japan \\ ${ }^{3}$ Collaborative Innovation Center of Extreme Optics, \\ Shanxi University, Taiyuan, \\ Shanxi 030006, China
}

*Electronic address: fyuewen@gmail.com (Y.-W.F); cgduan@clpm.ecnu.edu.cn (C.-G.D) 
Contents

I. The overall potential energy profile if $\delta_{1}>\delta_{2}$

II. The in-plane ferroelectricity in monolayer SnSe

III. The carrier concentration in doped $\mathrm{SnSe}$

IV. Phonon dispersions

V. Dopants with shallow levels

VI. Screening lengths of In:SnSe and Sb:SnSe electrodes

VII. Experimental feasibility for achieving doped SnSe

VIII. Depolarization field in In:SnSe/SnSe/Sb:SnSe

IX. Polar displacements profile

X. Layer-resolved density of states of In:SnSe/SnSe/Sb:SnSe

XI. The analysis of transmission eigenstate

XII. The width of extension layer in transport calculations

XIII. Hubbard $U$ effect on the TER effect 


\section{THE OVERALL POTENTIAL ENERGY PROFILE IF $\delta_{1}>\delta_{2}$}

Figure 1 shows the overall potential energy profiles if we assume $\delta_{1}>\delta_{2}\left(\delta_{1}\right.$ and $\delta_{2}$ are the screening lengths of left and right electrodes, respectively).

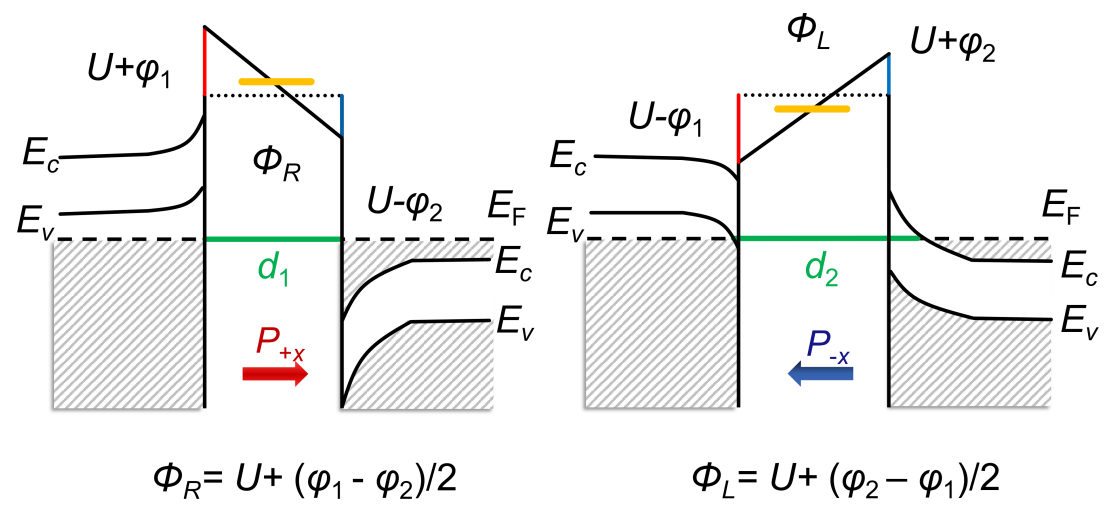

FIG. 1: Switching mechanisms of the 2D-FTJ $p$-SC/FE $/ n$-SC. The overall potential energy profiles with corresponding band diagrams if we assume $\delta_{1}>\delta_{2}$. Here, $\delta_{1}$ and $\delta_{2}$ are the screening lengths of left and right electrodes, respectively. The barrier width in either $P_{+x}$ or $P_{-x}$ state is given by the green line. The average potential barrier height is indicated by the short orange line. The Fermi level, as shown by black dashed line, is set as the reference of the barrier height.

\section{THE IN-PLANE FERROELECTRICITY IN MONOLAYER SNSE}

The top view of paraelectric SnSe monolayer $\left(P_{0}\right.$ state) is illustrated in Figure 2(a1), showing a high-symmetry lattice structure with the space group of $\mathrm{Cmcm}$. In this centrosymmetric $\mathrm{Cmcm}$ structure, the symmetry determines the atomic arrangements along $x$-axis and $y$-axis are exactly same. Hence, the corresponding noncentrosymmetric ferroelectric phase with polarization along $x$-axis (referred to as $P_{x}$ state) is exactly equivalent to the one with polarization along $y$-axis (referred to as $P_{y}$ state), as explicitly shown in Figure 2(a2) and (a3). We note that the armchair direction is always the direction of ferroelectric polarization. The space group of $P_{x}$ or $P_{y}$ state is Pnma. In the following sections and the main text, we only discuss $P_{x}$ state (i.e., along [100] direction) as an example.

Figure 2(b) shows the calculated double-well potential profile of the pure monolayer SnSe. In this pure phase without doping, the energy potential profile shows a symmetric feature, 
i.e., $P_{+x}$ and $P_{-x}$ are equivalent. The energy difference between the paraelectric and ferroelectric states is estimated to be $10.42 \mathrm{meV}$. The smooth energy curve connected paraelectric phase with the ferroelectric phase indicates the existence of a spontaneous symmetry breaking, in other words, the ferroelectric polarization is spontaneous.

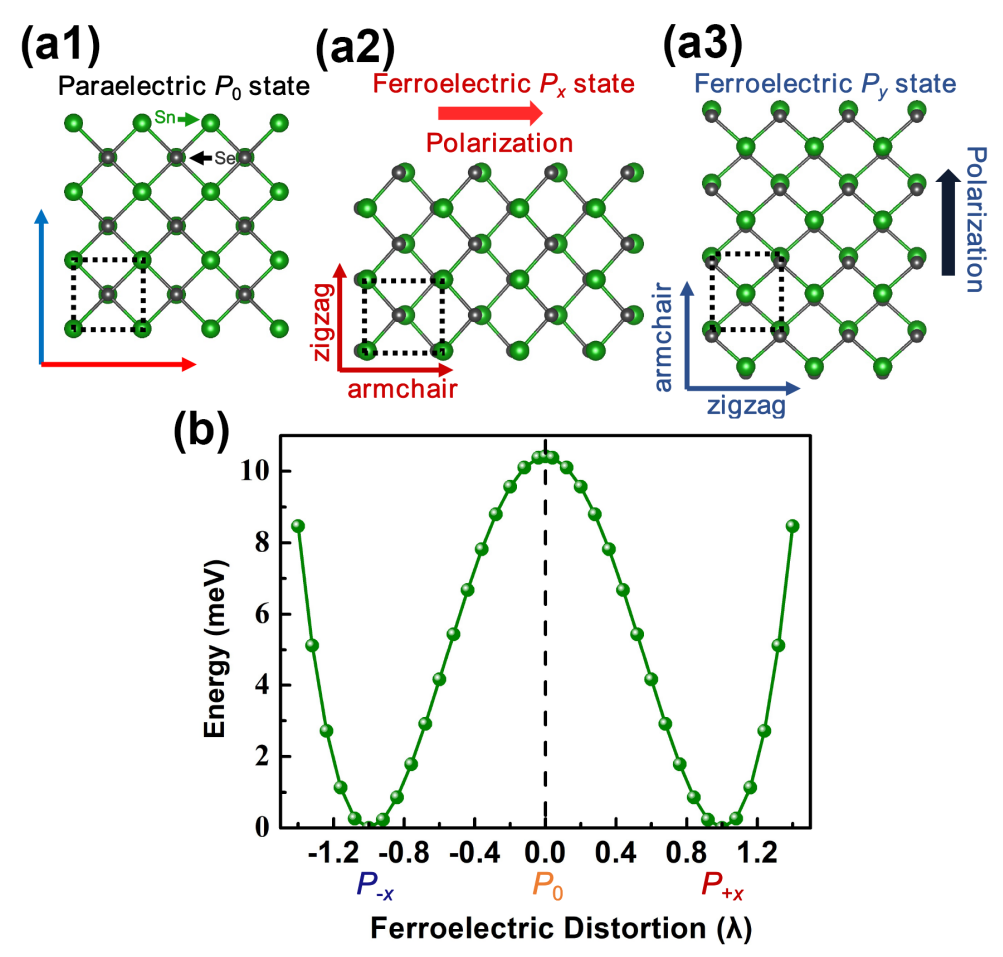

FIG. 2: The top views of lattice structure for the monolayer SnSe and the double well potential. (a1) The paraelectric state. (a2) The ferroelectric state along $x$-direction. (a3) The ferroelectric state along $y$-direction. The black dashed rectangle represents the primitive cell. (b) Calculated double-well potential energy profile of monolayer SnSe. The amplitude $\lambda$ of ferroelectric displacements along [100] directions is normalized, where $-1(+1)$ corresponds to the $P_{-x}\left(P_{+x}\right)$ state, and 0 corresponds to the paraelectric state.

\section{THE CARRIER CONCENTRATION IN DOPED SNSE}

In our calculations, the optimized lattice constants of the primitive cell for pristine SnSe are $\mathrm{a}=4.38 \AA, \mathrm{b}=4.29 \AA$, and $\mathrm{c}=21.46 \AA$, respectively. The unit cell size of electrode (i.e., doped SnSe) is a stacking of 4 unit-cells SnSe along $x$-axis in which one Sn atom is substituted by one In or Sb atom. Therefore, the size of unit cell doped SnSe is about 
$1612.95 \AA^{3}$, and the doping concentration can be calculated as $1 / 1612.95=6.20 \times 10^{20} \mathrm{~cm}^{-3}$.

\section{PHONON DISPERSIONS}

Figure 3(a) shows the phonon dispersion of paraelectric state of monolayer SnSe. Significant imaginary modes appearing at $\Gamma$ point are observed, indicating the large ferroelectric instability. The emerging unstable soft mode leads to the energy-lowering ferroelectric distortions of SnSe and the stabilization of ferroelectric state. The corresponding phonon dispersion of ferroelectric state is shown in Figure 3(b). By comparing our phonon calculations with that reported by Fei et al. [1], we find the phonon dispersions share similarities but also have minor differences. Specifically, we observe some differences of phonon modes around the high symmetry points of $\Gamma$ and $M$. The differences should mainly come from two reasons: 1) different lattice constants; 2) we note that the non-analytic contributions which are important for ferroelectric materials are not taken into consideration in Fei et al.'s study [1]. This leads to the slight difference in LO-TO splittings between their study and ours.
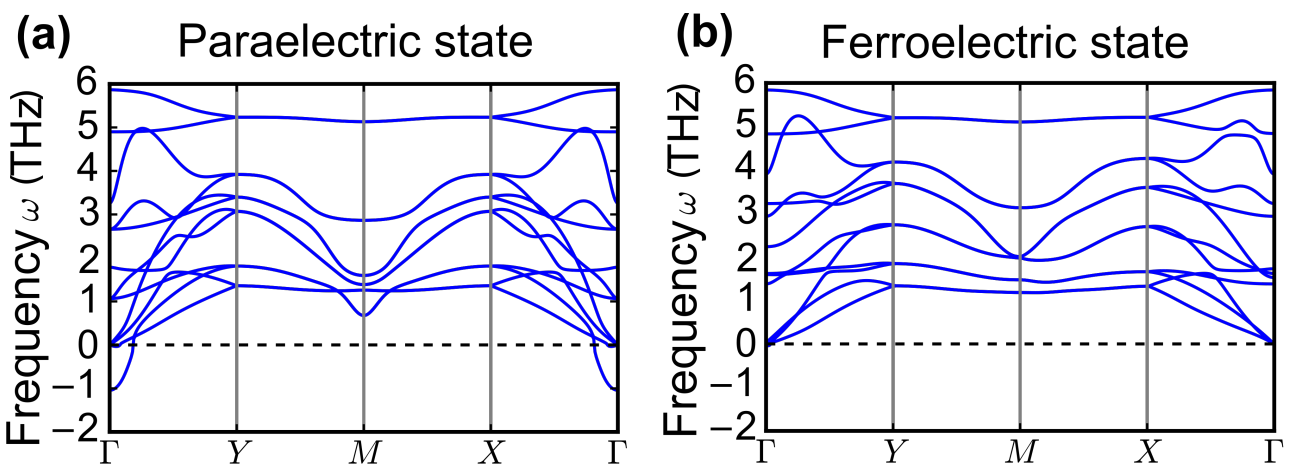

FIG. 3: Phonon dispersion curves along the high-symmetry $k$ path for the monolayer SnSe. (a) The paraelectric state with unstable mode. (b) The ferroelectric ground-state.

\section{DOPANTS WITH SHALLOW LEVELS}

Generally in the case of shallow donor or acceptor, the electronic states of defect introduced by the dopants will not be more than a few $k_{B} \mathrm{~T}$ below the conduction band minimum (CBM) or above the valence band maximum (VBM) edge, respectively [2]. In order to exam- 
ine the defect-induced levels in the doped $\mathrm{SnSe}$, we have calculated the electronic structures of monolayer In:SnSe and Sb:SnSe, the results are shown in Figure 4(a) and (b). For the $p$-type In:SnSe where In atoms act as the acceptors, the band structure and the corresponding density of states (DOS) show a heavily doped condition, in which VBM lies beyond the Fermi level. We show the weighted contributions of In dopants to electronic states using red solid circles, and find that the components of the defect-induced states appear predominantly near the VBM, therefore showing the key feature of a shallow acceptor level. In analogy to In:SnSe, we find Sb:SnSe also provides a shallow donor level. As a comparison, we show the total DOS for In:SnSe, Sb:SnSe, and SnSe in Figure 4(c).
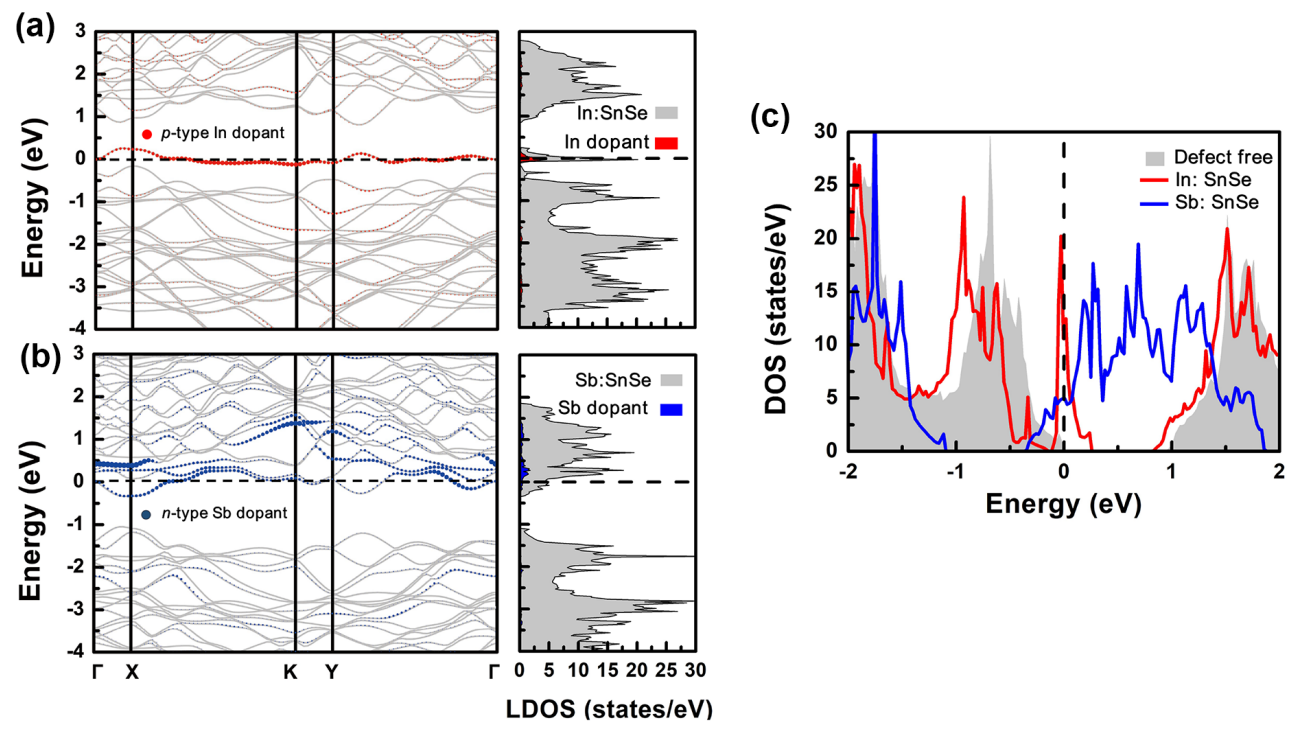

FIG. 4: Dopant-weighted band structure and the corresponding density of states (DOS) for (a) $p$-type In:SnSe and (b) n-type Sb:SnSe. The circles indicate the weighted contributions of dopants to the bands. (c) A comparison of the total DOS for In:SnSe, Sb:SnSe, and SnSe. The Fermi level is set at $0 \mathrm{eV}$.

\section{SCREENING LENGTHS OF IN:SNSE AND SB:SNSE ELECTRODES}

We estimate that the screening length of left electrode In:SnSe is smaller than the right electrode Sb:SnSe according to the Thomas-Fermi theory.

Specifically in Thomas-Fermi theory, the screening length of a metallic material is deter- 
mined by the materials' dielectric permittivity and electronic states:

$$
\delta=\frac{1}{e} \sqrt{\frac{\varepsilon}{\rho}}
$$

where $\varepsilon$ is the dielectric permittivity and $\rho$ is the density of states at the Fermi level $E_{\mathrm{F}}$. The dielectric permittivities of In:SnSe and $\mathrm{Sb}: \mathrm{SnSe}$ are equivalent to that of SnSe at saturation polarization [3]. Hence, the screening lengths of the two electrodes only depend on their densities of states at the $E_{\mathrm{F}}$ that can be easily controlled by doping. In our study, we only study the same $n$-type and $p$-type doping concentration, i.e., $6.2 \times 10^{20} \mathrm{~cm}^{-3}$ (see Section III). As shown in Figure 4, it can be found that the density of states of In:SnSe at the Fermi level is about two times of that of Sb:SnSe [4], indicating the screening length of In:SnSe is smaller than that of Sb:SnSe.

\section{EXPERIMENTAL FEASIBILITY FOR ACHIEVING DOPED SNSE}

According to Ref. [2], the formation energy of a defect in a charge state $q$ writes:

$$
\Delta H_{f}(\alpha, q)=E_{T}(\text { defect })-E_{T}(\text { perfect })+\sum_{\alpha} n_{\alpha}\left(\delta \mu_{\alpha}+\mu_{\alpha}^{\text {solid }}\right)+q\left(E_{v}+E_{\mathrm{F}}\right)
$$

where $E_{T}$ (defect) and $E_{T}$ (perfect) represent the total energies of the supercell with and without a defect, respectively. The third term of Equation 2 corresponds to the energy change due to exchange of atoms with the chemical reservoirs, where $\alpha$ determines which atom is added or removed for the defect. If an atom is added, $n_{\alpha}=-1$, while $n_{\alpha}=1$ if if an atom is replaced. $\delta \mu_{\alpha}$ is the elemental chemical potential of atom $\alpha$, referenced to the total ground-state solid $\mu_{\alpha}^{\text {solid }}$ of the pure elemental phase. The last term of Equation 2 is the energy change due to exchange of electrons and holes with the carrier reservoirs, in which $E_{v}$ is set to the energy at the valence-band maximum of the defect free system, and $E_{\mathrm{F}}$ is the Fermi energy of the system measured from the valence-band maximum.

For a neutral $(q=0)$ cation defect in the $p$-type doped left electrode, the formation energy with $n_{\mathrm{In}}=n_{\mathrm{Sn}}=1$ ( $i$.e., the case of one $\mathrm{Sn}$ atom replaced by $\mathrm{In}$ atom) can be calculated as following:

$$
\Delta H_{f}^{L}=E_{T}(\mathrm{In}: \mathrm{SnSe})-E_{T}(\mathrm{SnSe})-\mu_{\mathrm{In}}^{\mathrm{solid}}-\delta \mu_{\mathrm{In}}+\mu_{\mathrm{Sn}}^{\text {solid }}+\delta \mu_{\mathrm{Sn}}
$$


As a result, we can calculate the formation energy $H_{f}^{L}=0.90 \mathrm{eV}$. Similarly, for a neutral ( $q$ $=0)$ anion effect in the $n$-type doped right electrode, the formation energy is given by:

$$
\Delta H_{f}^{R}=E_{T}(\mathrm{Sb}: \mathrm{SnSe})-E_{T}(\mathrm{SnSe})-\mu_{\mathrm{Sb}}^{\text {solid }}-\delta \mu_{\mathrm{Sb}}+\mu_{\mathrm{Sn}}^{\text {solid }}+\delta \mu_{\mathrm{Sn}} .
$$

Thus, we get $H_{f}^{R}=1.48 \mathrm{eV}$.

In order to evaluate the feasibility of the doping, we make a comparison of the formation energy between the doped electrodes adopted here and previous works. For example, the native defects in single-layer $\mathrm{MoS}_{2}$ have been investigated in Ref. [5]. We can find that in the Mo-rich limit condition, the $\mathrm{S}$ vacancy $\left(V_{\mathrm{S}}\right)$ is found to be the most stable, of which the formation energy is only about $1.5 \mathrm{eV}$ for $V_{\mathrm{S}}^{0}$ and about $1.2 \mathrm{eV}$ for $V_{\mathrm{S}}^{1-}$. For the S-rich singlelayer $\mathrm{MoS}_{2}$, the $\mathrm{S}$ interstitial $\left(\mathrm{S}_{i}\right)$ is found the most stable with the formation energy only about $1.0 \mathrm{eV}$. Moreover, according to the stability of point defects in monolayer rhenium disulfide discussed in Ref. [6], we can find that the stable S-vacancy formation energies range from 1.16 to $2.39 \mathrm{eV}$ at the Re or S-rich condition. On account of these results, we can see that such formation energies for both left and right electrodes in our works confirm

the feasibility of doping, which is of great possibility to achieve In or Sb doped SnSe in experiment.

\section{DEPOLARIZATION FIELD IN IN:SNSE/SNSE/SB:SNSE}

We performed analysis of the electrostatic potential of the supercell that has been used in our double well potential calculations in the main text. The DFT-calculated electrostatic potential energy profiles for the $P_{+x}$ and $P_{-x}$ states are both shown in Figure 5. The asymmetry in potential profile can be observed, which is in agreement with the asymmetric features in the double-well potential profiles in our main text. The electrostatic potential energy presented in Figure 5 implies the contribution from the combination of (1) builtin electric field $E_{\mathrm{bi}}$ owing to the asymmetric interface, and (2) the depolarization field $E_{d}$ owning to the incomplete screening [7]. Different from $E_{\mathrm{bi}}$ which is invariant during the polarization reversal, $E_{\mathrm{d}}$ changes its direction because it is always opposite to the direction of ferroelectric polarization. By comparing the macroscopic average potential energy profiles, we find the slope in the SnSe regions in the $P_{+x}$ state (i.e., panel (a) of Figure 5) is larger than that in the $P_{-x}$ state, this implies $E_{\mathrm{bi}}$ and $E_{d}$ have the same direction in the $P_{+x}$ 
state, while they have opposite directions in the $P_{-x}$ state and result in smaller electric field. Specifically, we get

$$
\left\{\begin{array}{l}
E_{\mathrm{bi}}+E_{\mathrm{d}}=7.2 \times 10^{7} \mathrm{~V} / \mathrm{m} \\
E_{\mathrm{bi}}-E_{\mathrm{d}}=5.9 \times 10^{7} \mathrm{~V} / \mathrm{m} .
\end{array}\right.
$$

Therefore, $E_{\mathrm{bi}}$ and $E_{\mathrm{d}}$ are solved to be $6.6 \times 10^{7} \mathrm{~V} / \mathrm{m}$ and $6.5 \times 10^{6} \mathrm{~V} / \mathrm{m}$, respectively. The depolarization field in general ferroelectric films is around $10^{8} \sim 10^{9} \mathrm{~V} / \mathrm{m}[8,9]$, hence the very small depolarization field $\left(6.5 \times 10^{6} \mathrm{~V} / \mathrm{m}\right)$ in our study implies that the bound charge at the edges of ferroelectric regions can be well screened by the left/right electrodes. This indicates the ferroelectricity in the monolayer SnSe between the electrodes is very stable, which is in agreement with the double-well potential profile in Figure 3(b) in the main text.

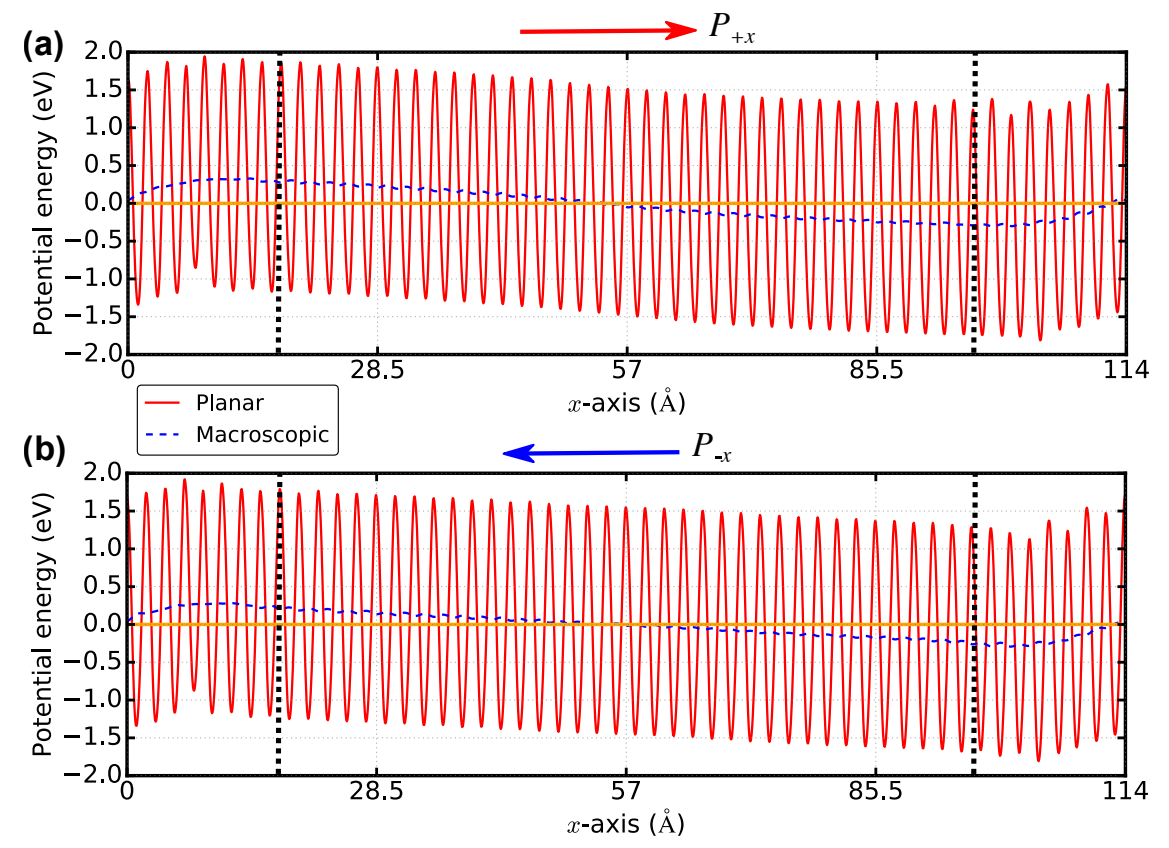

FIG. 5: The DFT-calculated electrostatic potential profiles of (a) $P_{+x}$ and (b) $P_{-x}$ states. The solid red curve and dashed blue curve refer to planar average and macroscopic average potentials, respectively. The vertical dashed black lines denote the interfaces.

\section{POLAR DISPLACEMENTS PROFILE}

Figure 6 shows the DFT-calculated polar displacements profile for both $P_{+x}$ and $P_{-x}$ states. 


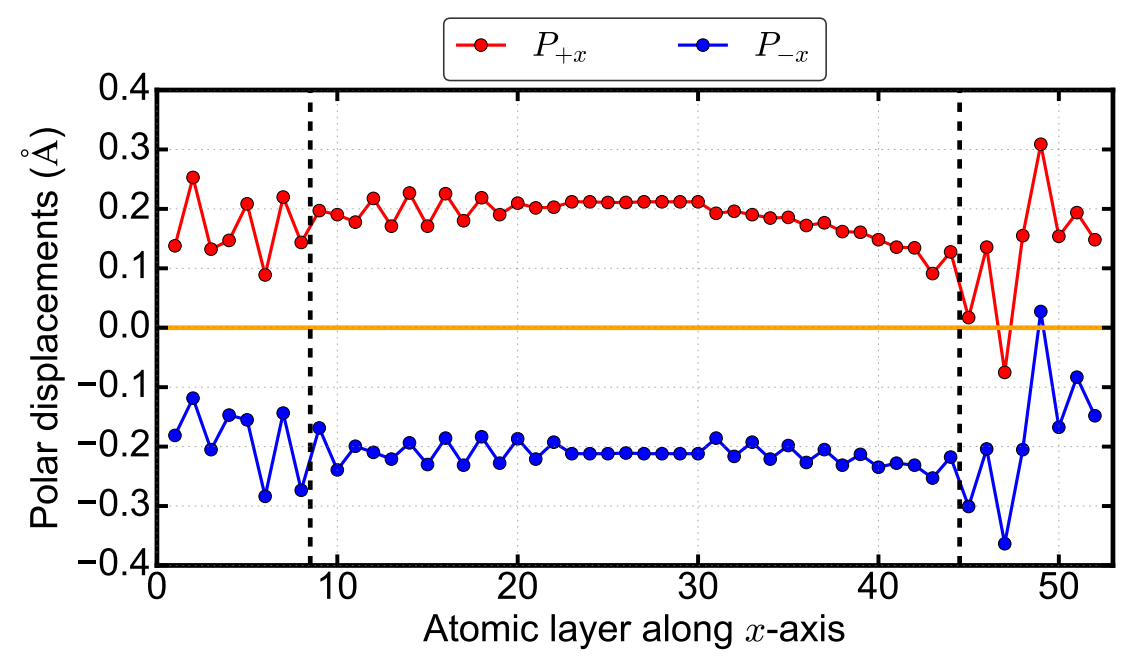

FIG. 6: The polar displacements profiles for $P_{+x}$ and $P_{-x}$ states of the In:SnSe/SnSe/Sb:SnSe junction. The measured polar displacements here refer to the displacements of cations with respect to the anions. The interfaces between the electrodes and barrier region are indicated by vertical dashed lines. 


\section{LAYER-RESOLVED DENSITY OF STATES OF IN:SNSE/SNSE/SB:SNSE}

Figure 7 shows the DFT-calculated layer-resolved density of states of In:SnSe/SnSe/Sb:SnSe junction, here layer in SnSe barriers refers to two unit cells along $x$-axis. In the In:SnSe (or $\mathrm{Sb}: \mathrm{SnSe}$ ) electrode, the densities of states contributed by all the unit cells are summed to make the demonstration clear. As shown in Figure 7, more SnSe unit cells in $P_{+x}$ state become metallic than $P_{-x}$ state. Consequently, the effective tunneling barrier width in $P_{-x}$ state is wider than $P_{+x}$ state.

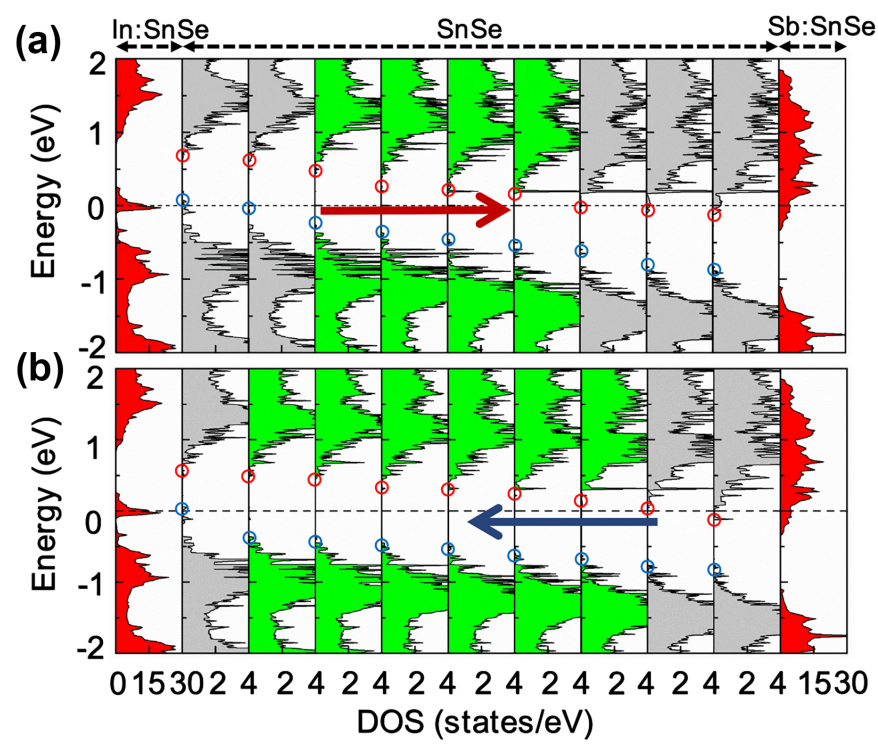

FIG. 7: Layer-resolved density of states for (a) $P_{+x}$ and (b) $P_{-x}$ state. The red and blue circles indicate the position of the CBM and VBM, respectively. The Fermi level is set to zero. The red shaded areas denote the electrode regions, the grey shaded areas represent the regions in $\mathrm{SnSe}$ barrier that become metallic, the green shaded ones correspond to the effective tunneling barrier.

\section{THE ANALYSIS OF TRANSMISSION EIGENSTATE}

We note that the transmission is mainly contributed by the region around $k_{y}=+0.42 /$ 0.42 instead of around $\Gamma$ point. In order to understand why the resonant tunneling occurs in these special $k$ points instead of $\Gamma$ point, we further study the transmission eigenstates at the Fermi level for $k=(0,-0.42)$ and $k=(0,0)$ (i.e., $\Gamma$ point). Since the transmission eigenstates correspond to the scattering state from the left electrode to the right electrode, 
we can find the electronic states that contribute to the electron transport.

Figure 8 shows the transmission eigenstates for the two studied $k$-points. By comparing Figure 8(a1) with (a2), or by comparing Figure 8(b1) with (b2), we can find the amplitude of transmission eigenstates in the middle to the right of the scattering region for $k=(0$, -0.42) is much larger than that for $\Gamma$ point, which indicates greater transmission probability (this can be read off from the eigenvalues shown in Figure 8) in the case of $k=(0,-0.42)$ in respective $P_{+x}$ and $P_{-x}$ states. This is particularly obvious in $P_{+x}$ state. We also find the tranmission eigenstate for $k=(0,+0.42)$ is similar to that for $k=(0,-0.42)$, which is not shown here. The difference of the transmission eigenstates rises from the different decay rates of the states at these $k$-points. For both $k=(0,-0.42)$ and $\Gamma$ point, the transmission eigenstate decays through the tunneling barrier. However, the decay rate for $k=(0,-0.42)$ is much smaller than that for $\Gamma$ point, i.e., the effective transmission channel is suppressed around $\Gamma$ point. This explains why the resonant tunneling mainly occurs in the region around $k_{y}=+0.42 /-0.42$, but not the $\Gamma$ point.

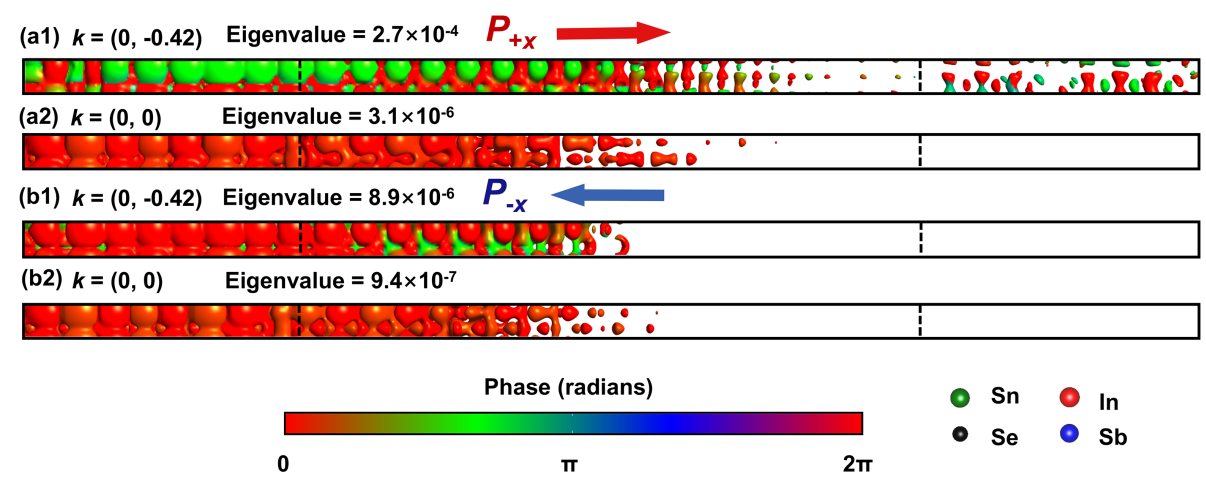

FIG. 8: Caption continues on the next page.

\section{THE WIDTH OF EXTENSION LAYER IN TRANSPORT CALCULATIONS}

In DFT+NEGF calculations, the extension layer (buffer layer) should be large enough to screen the electrostatic potential. In our study, the left/right extension layer is as wide as around $35 \AA$, which is almost equivalent to the width of 8 u.c. SnSe. Figure 9 shows the planar average (solid red curve) and macroscopic average (dashed blue curve) electrostatic potential energy differences obtained in our DFT+NEGF calculations. We can find that the macroscopic average potential differences are nearly flat for both left and right polar- 
FIG. 8: The transmission eigenstates in the central region at the Fermi level for $k=(0,-0.42)$ and $k=(0,0)$. Here, the central region is consisted of the left/right extension layer (buffer layer, 2 u.c. doped SnSe) and the ferroelectric barrier layer (as thick as 18 u.c. SnSe). (a) For $P_{+x}$ state: (a1) $k=(0,-0.42)$; (a2) $k=(0,0)$. (b) For $P_{-x}$ state: (b1) $k=(0,-0.42)$; (b2) $k=(0,0)$. In all the panels, we use same isovalue for comparison. The color bar indicates the phase of the eigenstate. The vertical dashed black lines denote the interfaces between the ferroelectric SnSe and the buffer layers.

ization states, which indicates our extension layer is large enough to screen the electrostatic potential.
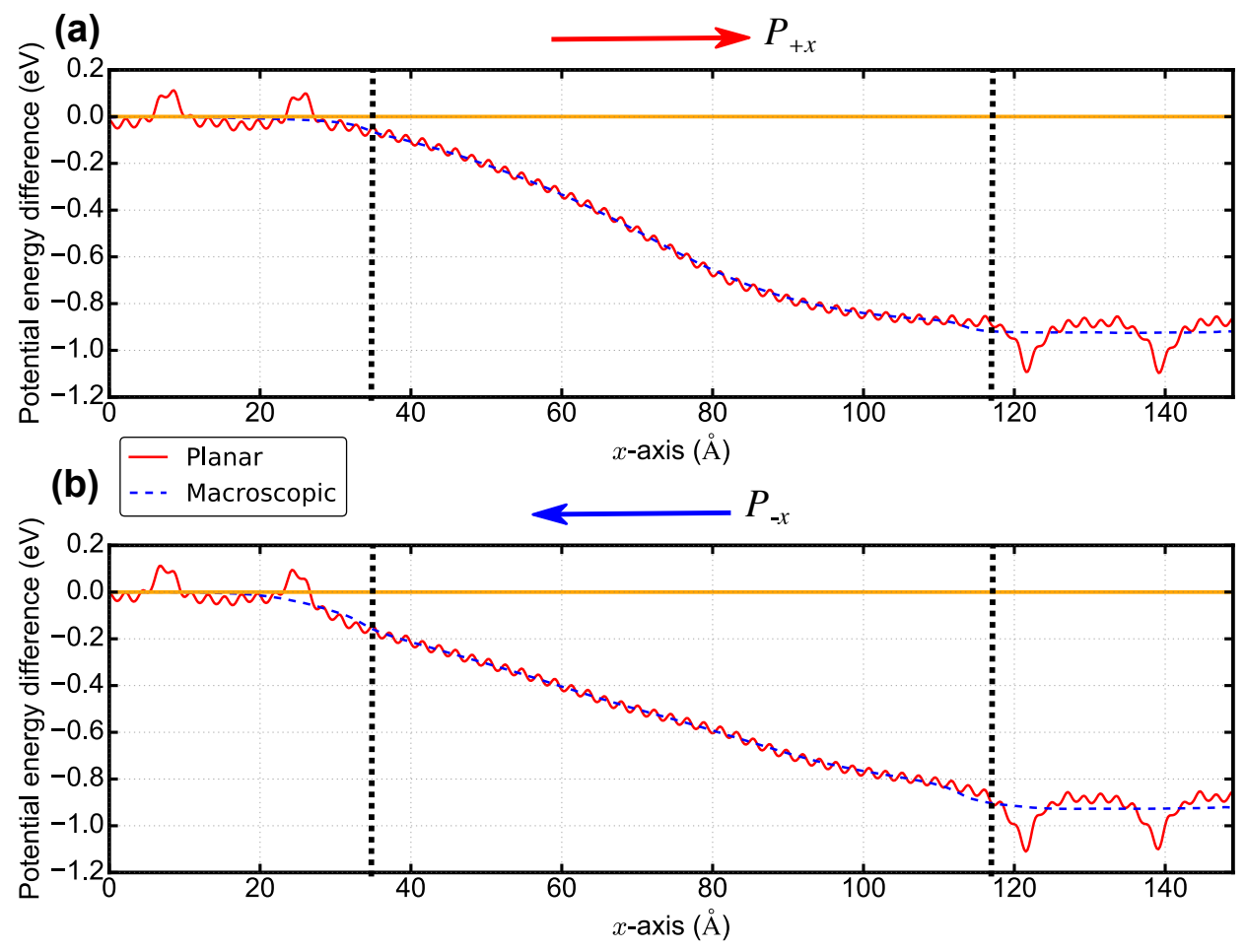

FIG. 9: Caption continues on the next page.

\section{HUBBARD $U$ EFFECT ON THE TER EFFECT}

In Ref. [10], Hubbard $U=4 \mathrm{eV}$ is used to reproduce the band gaps of Sn-based ferroelectric semiconductors, the reported band gaps are in good agreement with the exprimental values. Here, in order to have a better estimation of the band gap of SnSe, we also introduce 
FIG. 9: The electrostatic potential energy differences between the electrostatic potential of the self-consistent valence charge density (the solution to the Poisson equation with this density) and the electrostatic potential from a superposition of atomic valence densities for (a) $P_{+x}$ and (b) $P_{-x}$ states obtained in our DFT+NEGF calculations. The solid red curve and dashed blue curve refer to planar average and macroscopic average potential energy differences, respectively. The vertical dashed black lines denote the interfaces between the ferroelectric SnSe and the buffer layers.

Hubbard $U=4 \mathrm{eV}$ into our DFT and DFT+NEGF calculations. The Hubbard $U$ is applied to the $p$ orbitals of Sn. Figure 10 shows the comparison of band gaps of SnSe between DFT and DFT $+U$ calculations. In DFT and DFT $+U$ calculations, the band gaps of SnSe are 0.9 and $1.4 \mathrm{eV}$, respectively. Compared to the experimental band gap ( $\sim 1.6 \mathrm{eV})$ of monolayer SnSe [11], DFT $+U$ study shows better estimation. We thus revisit the electron transport properties of 2D-FTJ In:SnSe/SnSe/Sb:SnSe homostructure by DFT+U+NEGF $(U=4$ eV)calculations. The calculated TER is $\sim 1120 \%$. In our main text, the DFT+NEGF calculated TER is $\sim 1460 \%$. We can find the order magnitude is not changed by the included Hubbard $U$. Hence, we think the TER of our studied 2D-FTJ is weakly affected by the estimation of band gap of SnSe. In addition, we note that the correction of band gap of the ferroelectric barrier material in the conventional FTJ $\mathrm{SrRuO}_{3} / \mathrm{PbTiO}_{3} / \mathrm{Pt}$ junction reported by Tao et al. [12] is also found to have minor effect on the main results of tunneling properties. This implies DFT+NEGF calculations can give very good estimation of the transport properties in FTJs.

[1] R. Fei, W. Kang, and L. Yang, Phys. Rev. Lett. 117, 097601 (2016).

[2] C. Freysoldt, B. Grabowski, T. Hickel, J. Neugebauer, G. Kresse, A. Janotti, and C. G. Van de Walle, Rev. Mod. Phys. 86, 253 (2014).

[3] Y. Wang, X. Liu, J. D. Burton, S. S. Jaswal, and E. Y. Tsymbal, Phys. Rev. Lett. 109, 247601 (2012).

[4] See the Supporting Information for more details.

[5] J.-Y. Noh, H. Kim, and Y.-S. Kim, Phys. Rev. B 89, 205417 (2014).

[6] S. Horzum, D. Çak ır, J. Suh, S. Tongay, Y.-S. Huang, C.-H. Ho, J. Wu, H. Sahin, and F. M. 


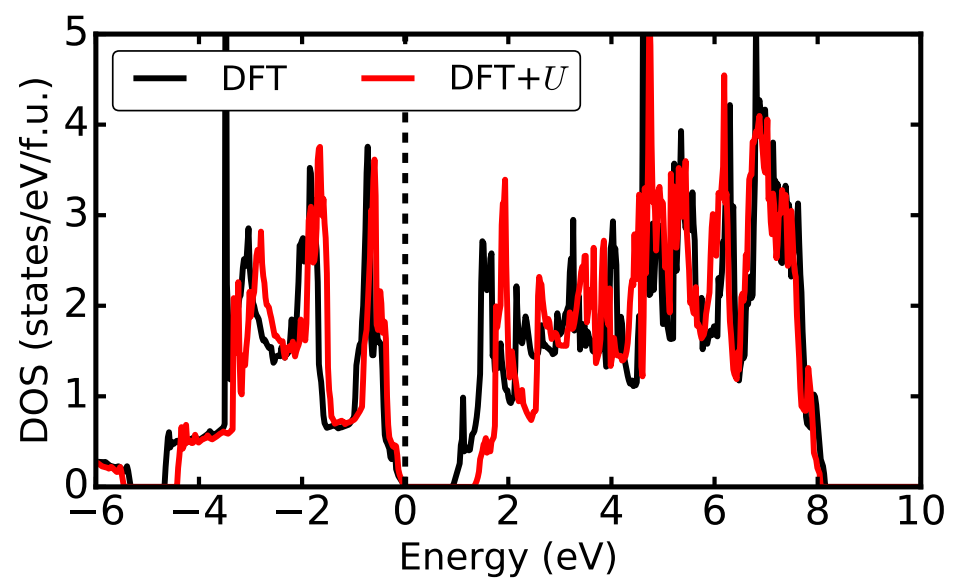

FIG. 10: The total density of states of SnSe which is normalized to per formula unit. The black and red curves refer to DFT and DFT $+U(U=4 \mathrm{eV})$ calculated DOS, respectively. The dashed vertical line indicate the Fermi level.

Peeters, Phys. Rev. B 89, 155433 (2014).

[7] X. Luo, Y. Zheng, and B. Wang, J. Appl. Phys. 111, 074102 (2012).

[8] S. B. Lang and H. L. Chan, Frontiers of ferroelectricity: a special issue of the journal of materials science (Springer Science \& Business Media, 2007).

[9] C. P. De Araujo, J. F. Scott, and G. W. Taylor, Ferroelectric thin films: synthesis and basic properties, vol. 10 (Taylor \& Francis US, 1996).

[10] T. Babuka, K. Glukhov, Y. Vysochanskii, and M. Makowska-Janusik, RSC Adv. 7, 27770 (2017).

[11] W. Shi, M. Gao, J. Wei, J. Gao, C. Fan, E. Ashalley, H. Li, and Z. Wang, Adv. Sci. 5, 1700602 (2018).

[12] L. L. Tao and J. Wang, J. Appl. Phys. 119, 224104 (2016). 